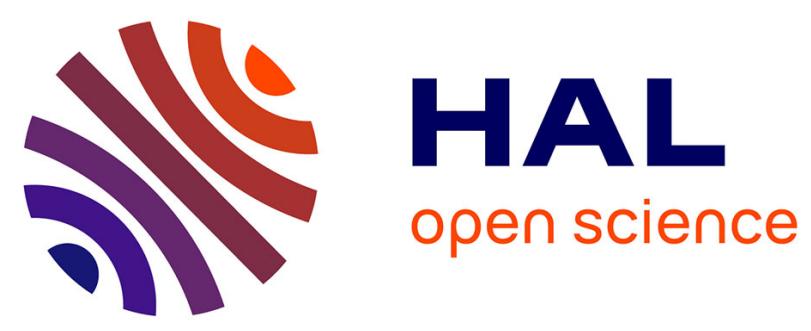

\title{
Symbolic and numeric scheme for solution of linear integro-differential equations with random parameter uncertainties and Gaussian stochastic process input
}

Igor E. Poloskov, Christian Soize

\section{- To cite this version:}

Igor E. Poloskov, Christian Soize. Symbolic and numeric scheme for solution of linear integrodifferential equations with random parameter uncertainties and Gaussian stochastic process input. Applied Mathematical Modelling, 2018, 56, pp.15-31. 10.1016/j.apm.2017.11.024 . hal-01655300

\author{
HAL Id: hal-01655300 \\ https://hal.science/hal-01655300
}

Submitted on 4 Dec 2017

HAL is a multi-disciplinary open access archive for the deposit and dissemination of scientific research documents, whether they are published or not. The documents may come from teaching and research institutions in France or abroad, or from public or private research centers.
L'archive ouverte pluridisciplinaire HAL, est destinée au dépôt et à la diffusion de documents scientifiques de niveau recherche, publiés ou non, émanant des établissements d'enseignement et de recherche français ou étrangers, des laboratoires publics ou privés. 


\title{
Symbolic and numeric scheme for solution of linear integro-differential equations with random parameter uncertainties and Gaussian stochastic process input
}

\author{
I. E. Poloskov ${ }^{\mathrm{a}, *}$, C. Soize ${ }^{\mathrm{b}}$ \\ ${ }^{a}$ Faculty of Mechanics and Mathematics, Perm State University, 15 Bukirev street, 614990 Perm, \\ GSP, Russia \\ ${ }^{b}$ Laboratoire Modélisation et Simulation Multi Echelle, MSME UMR 8208 CNRS, 5 bd \\ Descartes, 77454 Marne-la-Valle, France
}

\begin{abstract}
The paper describes a theoretical apparatus and an algorithmic part of application of the Green matrix-valued functions for time-domain analysis of systems of linear stochastic integro-differential equations. It is suggested that these systems are subjected to Gaussian nonstationary stochastic noises in the presence of model parameter uncertainties that are described in the framework of the probability theory. If the uncertain model parameter is fixed to a given value, then a time-history of the system will be fully represented by a second-order Gaussian vector stochastic process whose properties are completely defined by its conditional vector-valued mean function and matrix-valued covariance function. The scheme that is proposed is constituted of a combination of two subschemes. The first one explicitly defines closed relations for symbolic and numeric computations of the conditional mean and covariance functions, and the second one calculates unconditional characteristics by the Monte Carlo method. A full scheme realized on the base of Wolfram Mathematica and Intel Fortran software programs, is demonstrated by an example devoted to an estimation of a nonstationary stochastic response of a mechanical system with a thermoviscoelastic component. Results obtained by using the proposed scheme are compared with a reference solution constructed by using a direct Monte Carlo simulation.
\end{abstract}

Keywords: stochastic integro-differential equation, random parameter, Gaussian input, Green function, second-order moments' calculation, Monte Carlo simulation

\footnotetext{
${ }^{*}$ Corresponding author

Email addresses: igor.poloskovepsu.ru (I. E. Poloskov), christian.soize@univ-paris-est.fr(C. Soize)
} 
2010 MSC: 11K45, 60H35, 65C30, 74D05

\section{Introduction}

\subsection{Subject area, models, review of tools, and structure of the paper}

Deterministic ordinary integral differential equations (OIDE) and stochastic ordinary integral differential equations (SOIDE) are interesting from both academic scientific and technical points of view because these equations are models of phenomena in a huge number of different sectors. A common theory and primary classification of deterministic partial integral differential equations (PIDE) was developed by Vito Volterra in the first half of twentieth century. General ideas of stochastic dynamics have been considered, for instance, in [1, 2, 3, 4, 5, 6].

In many cases, sources of models in the form of SOIDE in stochastic mechanics are results of space discretizations of stochastic partial integro-differential equations (SPIDE) that describe continuous viscoelastic media [7]. Techniques of such discretizations are usually based on well-known ideas of the finite element method, the finite difference method and another computation schemes for solution of space-time problems.

The first investigations of viscoelastic behavior were made by W.E. Weber, R. and F. Kohlrausch, L. Boltzmann, O. Meyer, D.K. Maxwell, W. Thomson (Lord Kelvin), W. Voigt, P. Duhem, L. Natanson, S. Zaremba, A.E. Green and R.S. Rivlin (1957), B.D. Coleman and W. Noll (1958, 1961, 1964), mainly in the second half of nineteenth century. Historical remarks about developments in the viscoelastic domain can be found in [8].

Foundations of modern theory of viscoelasticity were expounded in [8]. An important part of this theory is an apparatus for investigation of time-nonhomogeneous systems [9]. In the case of linear aging viscoelasticity, where the term aging means that the mechanical properties of a given material are changed with its age, the constitutive equations for linear viscoelastic media with infinitesimal strains can be expressed as Stieltjes integrals (according to Riesz's representation theorem [8]), and are written as

$$
\begin{array}{cc}
\sigma_{i j}(r, t)=\sum_{k, \ell=1}^{3} \int_{t_{0}}^{t} F_{i j k \ell}(r, t, \tau) d \varepsilon_{k \ell}(r, \tau), & \sigma_{i j}\left(r, t_{0}\right)=0, \\
\varepsilon_{i j}(r, t)=\sum_{k, \ell=1}^{3} \int_{t_{0}}^{t} G_{i j k \ell}(r, t, \tau) d \sigma_{k \ell}(r, \tau), & \varepsilon_{i j}\left(r, t_{0}\right)=0, \\
\varepsilon_{i j}(r, t)=\frac{1}{2}\left[\frac{\partial u_{i}(r, t)}{\partial x_{j}}+\frac{\partial u_{j}(r, t)}{\partial x_{i}}\right], & i, j=1,2,3,
\end{array}
$$


where $t$ is a current time, $t_{0} \leqslant t \leqslant T<+\infty, r=\left(x_{1}, x_{2}, x_{3}\right)$ is the vector of material (Lagrangian) coordinates of a material point of the system under investigation, $u(r, t)=\left\{u_{i}(r, t)\right\}$ is the vector of spatial displacements of material points as a function of the reference position, $r$, and $t, \epsilon(t)=\left\{\varepsilon_{i j}(r, t)\right\}$ is the strain tensor, and $\sigma(r, t)=\left\{\sigma_{i j}(r, t)\right\}$ is the stress tensor. Correspondingly, $F_{i j k \ell}(r, t, \tau)$ is the tensorial creep function and $G_{i j k \ell}(r, t, \tau)$ is the tensorial relaxation function. These kernels of integral operators depend on $t$ and $\tau$ separately but not on difference $t-\tau$ and describe a relationship of the current stress with the whole time history of strain. After transformation of PIDE or SPIDE into OIDE or SOIDE, a time-dependent structure of the kernels is preserved in target equations of motion.

An important part of studies in stochastic viscoelasticity includes: (i) an analysis of a weak solvability of initial-boundary value problems [10], (ii) an examination of a stochastic stability [11,9], (iii) solution of reliability problems, etc.

It is well-known, that the solution of integral differential equations (IDE) is a very difficult problem even for the deterministic case. These difficulties are even greater for the linear and nonlinear stochastic cases. In spite of the existence of a few results concerning solvers for SOIDE (see for instance, [7]), it is very useful to adapt the existing methods for solving deterministic IDE to the stochastic case, because the main part of methods for qualitative and quantitative analysis of phenomena described by SOIDE consists of deterministic schemes.

A number of schemes have been developed for constructing numerical approximations for solution of deterministic and stochastic IDE. As for examination of stochastic problems, approximate algorithms are usually used for direct generation of time histories. Among these schemes there are:

(i) purely numerical methods (classical and modified hybrid variants) such as explicit and implicit (backward) Euler schemes, a discontinuous Galerkin method, energy methods [12], general, standard and Galerkin finite element methods for PIDE, the Tau method [13], the one-step Runge-Kutta and multi-step methods $[14,15,16]$, the Runge-Kutta method [17] for calculation of covariance functions, extrapolation methods [18], Galerkin methods [19], the method of iterations at the last step, a usage of wavelets [20], globally defined Sinc basis functions [21], an approximate transformation of SOIDE into SODE on the base of replacements of kernels with respect to second arguments by piecewise constant functions [22, 7], and gamma-distribution expansions;

(ii) approximate analytic methods, including methods of Taylor series [23], the successive approximation method for the computation of the Green function [24], the asymptotic method [25], the stochastic averaging method, the collocation method [26], the perturbation theory [27].

The main objective of this paper is to present a new tool for a statistical estimation of the solution of a class of IDE with random parameters and Gaussian stochastic processes as input. Stochastic linear viscoelastic problems are de- 
scribed by this class. The paper explains a theoretical apparatus and an algorithmic part of an application of the Green matrix-valued functions for time-domain analysis of systems of linear SOIDE effected by Gaussian nonstationary noises in the presence of model parameter uncertainties that are described in the framework of the probability theory.

This paper is organized as follows. Section 2 deals with the formulation of the problem under investigation. Section 3 is devoted to the construction of the equations for calculating the first- and second-order conditional moment functions of the stochastic solution. In Section 4, we give a short review of an application of the Green matrix-valued function for calculation of solution of linear IDE, and schemes for its approximate computation. Section 5 yields relations for Green matrix-valued functions for the first- and second-order conditional moment functions, and formal schemes for calculation of the conditional vector-valued mean function and the conditional matrix-valued covariance function. Section 6 deals with some approximations for the first- and second-order conditional moment functions obtained on the base of Green matrix-valued functions. In Section 7, we present a stochastic solver that produces the first- and second-order unconditional moment functions on the base of the first- and second-order conditional moment functions. Section 8 deals with an application devoted to a linear secondorder dynamical system with a thermoviscoelastic component. We conclude the paper with Section 9. Appendix A consists of a few Green function components.

\subsection{Comments concerning notation used}

In this paper, the following notations are used:

(1) lower case letters for real deterministic variables and vectors (e.g. $x, x=$ $\left.\left.\left(x_{1}, \ldots, x_{n}\right)\right), f, f=\left(f_{1}, \ldots, f_{n}\right)\right)$;

(2) upper case letters for real random variables and vectors (e.g. $X, X=$ $\left.\left(X_{1}, \ldots, X_{n}\right)\right)$

(3) double lined upper case letters for sets or spaces (e.g. $\mathbb{R}$ ).

(4) upper case letters and script (calligraphic) math upper case letters (the Zapf Chancery font) for real deterministic matrices (e.g. $A, A=\left\{a_{i j}\right\}, \mathcal{A}, \mathcal{A}=\left\{a_{i j}\right\}$ );

(5) calligraphic upper case letters for real random matrices (e.g. $\mathcal{A}=\left\{\mathcal{A}_{i j}\right\}$ );

(6) any deterministic quantity above (e.g. $x, A$ ) with an underline (e.g. $\underline{x}$, $\underline{A}$ ) means that this deterministic quantity is related to the mean model (or to the nominal model).

\section{Formulation of the problem}

In this paper, the following system of stochastic integro-differential equations is considered, 


$$
\begin{array}{r}
\dot{X}(t)=\mathcal{A}(t ; Q) X(t)+\int_{t_{0}}^{t} \mathcal{B}(t, \tau ; Q) X(\tau) d \tau+\mathcal{G}(t ; Q)[V(t)+v(t)], \\
t_{0}<t \leqslant T, \quad X\left(t_{0}\right)=X_{0},
\end{array}
$$

where $t$ is time and $t_{0}$ is the initial time such that $t_{0}<T<+\infty$. An upper dot denotes the symbol of derivative with respect to $t$. The vector $X(t)=$ $\left(X_{1}(t), \ldots, X_{n}(t)\right)$ is the state vector in $\mathbb{R}^{n}$. The initial condition $X_{0}$ is a random vector and the input $V$ is a stochastic process which is independent of $X_{0}$.

The vector $X_{0}$ is a Gaussian second-order random variable with values in $\mathbb{R}^{n}$. Its mean value is $m_{X_{0}}=\mathbf{E}\left\{X_{0}\right\}$ and its covariance matrix is $C_{X_{0} X_{0}}=$ $\mathbf{E}\left\{\left(X_{0}-m_{X_{0}}\right)\left(X_{0}-m_{X_{0}}\right)^{\top}\right\}$ which is assumed to be invertible. In this paper, $\mathbf{E}\{\}$ denotes the mathematical expectation. The input $\left\{V(t)=\left(V_{1}(t), \ldots\right.\right.$, $\left.\left.V_{m}(t)\right), t \geqslant t_{0}\right\}$ is a stochastic process with values in $\mathbb{R}^{m}$, which is governed by the linear Itô stochastic differential equation,

$$
d V(t)=H V(t) d t+R d W(t), \quad t>t_{0}, \quad V\left(t_{0}\right)=V_{0},
$$

where $V_{0}$ is a vector random variable. In Eqs. (5), $H$ and $R$ are deterministic real matrices in $\mathbb{R}^{m \times m}$ and $\mathbb{R}^{m \times r}$ respectively, $\left\{W(t)=\left(W_{1}(t), \ldots, W_{r}(t)\right), t \geqslant t_{0}\right\}$ is the $\mathbb{R}^{r}$-valued Wiener stochastic process with independent components. Its generalized derivative with respect to $t$, denoted by $\left\{\dot{W}(t)=\left(\dot{W}_{1}(t), \ldots, \dot{W}_{r}(t)\right)\right.$, $\left.t \geqslant t_{0}\right\}$, is the vector Gaussian white noise with independent components such that

$$
\mathbf{E}\{\dot{W}(t)\}=0, \quad \mathbf{E}\left\{\dot{W}(t) \dot{W}^{\top}\left(t^{\prime}\right)\right\}=2 \pi E \delta_{0}\left(t-t^{\prime}\right) .
$$

In Eqs. (6), $E$ is the identity matrix in $\mathbb{R}^{r \times r}$ and $\delta_{0}$ is the Dirac generalized function at 0 in $\mathbb{R}$.

The initial vector $V_{0}$ is a Gaussian, second-order, centered random variable with values in $\mathbb{R}^{m}$. Its probability distribution is the Gaussian invariant measure associated with Eqs. (5) such that the mean value is $m_{V_{0}}=\mathbf{E}\left\{V_{0}\right\}=0$ and the covariance matrix is $C_{V_{0} V_{0}}=\mathbf{E}\left\{V_{0} V_{0}^{\top}\right\}$. In Eqs. (5), matrices $H$ and $R$ are assumed to be such that $V$ is a second-order stochastic process. Consequently, $\left\{V(t), t \geqslant t_{0}\right\}$ is a Gaussian, stationary (for the right translation), second-order, centered and mean-square continuous stochastic process such that the mean function and the covariance function are

$$
\begin{gathered}
m_{V}(t)=\mathbf{E}\{V(t)\}=m_{V_{0}}=0, \\
C_{V V}\left(t_{1}-t_{2}\right)=C_{V V}\left(t_{1}, t_{2}\right)=\mathbf{E}\left\{V\left(t_{1}\right) V^{\top}\left(t_{2}\right)\right\}, \quad C_{V V}(0)=C_{V_{0} V_{0}} .
\end{gathered}
$$

In Eqs. (4), $Q=\left(Q_{1}, \ldots, Q_{s}\right)$ is a random vector with values in $\mathbb{D}_{Q} \subset \mathbb{R}^{s}$ for which its probability distribution $\mathrm{P}_{Q}(d q)$ is given. Matrices $\mathcal{A}(\cdot ; \cdot) \in \mathbb{R}^{n \times n}$, 
$\mathcal{B}(\cdot, \cdot ; \cdot) \in \mathbb{R}^{n \times n}$, and $\mathcal{G}(\cdot, \cdot ; \cdot) \in \mathbb{R}^{n \times m}$ depend on $Q$ and consequently, are random matrices. For $Q$ fixed to $q=\left(q_{1}, \ldots, q_{s}\right)$ in $\mathbb{R}^{s}$, and for all $t$ and $\tau$ fixed such that $t_{0}<\tau \leqslant t \leqslant T, A(t ; q), B(t, \tau ; q)$, and $G(t ; q)$ are deterministic matrices.

The given deterministic function $t \mapsto v(t)=\left(v_{1}(t), \ldots, v_{m}(t)\right)$ with values in $\mathbb{R}^{m}$ is assumed to be continuous. For all $q$ fixed in $\mathbb{D}_{Q}$, the matrix-valued functions $t \mapsto A(t ; q)$ and $t \mapsto B(t, \tau ; q)$ are assumed to be continuous and piecewise differentiable until necessary order, and the matrix-valued function $t \mapsto G(t ; q)$ is assumed to be continuous.

For all $Q=q$ fixed in $\mathbb{D}_{Q}$, the stochastic process $X$ is rewritten as $X^{Q}$ and the notation $X^{Q}$ means that we consider stochastic process $X$ for given $Q$. Since Eqs. (4) are linear, the vector $X_{0}$ and the stochastic process $V$ are Gaussian because of the introduced hypotheses, it can easily be proved that stochastic process $\left\{X^{Q}(t), t \in\left[t_{0}, T\right]\right\}$ is a Gaussian, second-order and mean-square continuous stochastic process.

For all finite nonempty and ordered subset $t=\left\{t_{1}, t_{2}, \ldots, t_{p}\right\}$ of $\left[t_{0}, T\right]$, the $p$-dimensional conditional probability distribution, $\mathrm{P}_{X^{Q}}(d \chi ; t \mid Q)$, given $Q$, of the random vector $X^{Q}=\left(X^{Q}\left(t_{1}\right), \ldots, X^{Q}\left(t_{p}\right)\right)$, is a Gaussian measure that is entirely defined by its mean vector,

$$
m_{X}^{Q}(t)=\left(m_{X}^{Q}\left(t_{1}\right), \ldots, m_{X}^{Q}\left(t_{p}\right)\right) \quad \text { with } \quad m_{X}^{Q}\left(t_{i}\right)=\mathbf{E}\left\{X^{Q}\left(t_{i}\right)\right\},
$$

and its covariance matrix $\mathcal{C}_{X}^{Q}(t)=\left\{\mathcal{C}_{X}^{Q}\left(t_{i}, t_{j}\right)\right\}$ such that, for all $i$ and $j$ in $\{1, \ldots, p\}$,

$$
\mathcal{C}_{X}^{Q}\left(t_{i}, t_{j}\right)=\mathbf{E}\left\{\left\{X^{Q}\left(t_{i}\right)-m_{X}^{Q}\left(t_{i}\right)\right\}\left\{X^{Q}\left(t_{j}\right)-m_{X}^{Q}\left(t_{j}\right)\right\}^{\top}\right\} .
$$

Consequently, the non-Gaussian probability distribution, $\mathrm{P}_{X}(d \chi ; t)$, of the random vector $\mathcal{X}=\left(X\left(t_{1}\right), \ldots, X\left(t_{p}\right)\right)$ can be calculated by

$$
\mathrm{P}_{X}(d \chi ; t)=\int_{\mathbb{D}_{Q}} \mathrm{P}_{X Q}(d \chi ; t \mid q) \mathrm{P}_{Q}(d q) .
$$

Let us now define the mean functions and the covariance functions of the stochastic process $X$ and the stochastic process $X^{Q}$ for given $Q$, together with the cross-covariance functions of $X$ and $X^{Q}$ with the stochastic process $V$ :

$$
\begin{gathered}
m_{X}(t)=\mathbf{E}\{X(t)\}, \quad m_{X}^{Q}(t)=\mathbf{E}\left\{X^{Q}(t)\right\}, \\
C_{X X}\left(t_{1}, t_{2}\right)=\mathbf{E}\left\{X\left(t_{1}\right) X^{\top}\left(t_{2}\right)\right\}-m_{X}\left(t_{1}\right) m_{X}^{\top}\left(t_{2}\right), \\
\mathcal{C}_{X X}^{Q}\left(t_{1}, t_{2}\right)=\mathbf{E}\left\{X^{Q}\left(t_{1}\right) X^{Q \top}\left(t_{2}\right)\right\}-m_{X}^{Q}\left(t_{1}\right) m_{X}^{Q \top}\left(t_{2}\right), \\
C_{X V}\left(t_{1}, t_{2}\right)=\mathbf{E}\left\{X\left(t_{1}\right) V^{\top}\left(t_{2}\right)\right\}, \quad \mathcal{C}_{X V}^{Q}\left(t_{1}, t_{2}\right)=\mathbf{E}\left\{X^{Q}\left(t_{1}\right) V^{\top}\left(t_{2}\right)\right\} .
\end{gathered}
$$


In Eqs. (8), for fixed $Q=q$ in $\mathbb{D}_{Q}$, the conditional moments $m_{X}^{Q}(t), \mathcal{C}_{X X}^{Q}\left(t_{1}, t_{2}\right)$ and $\mathcal{C}_{X V}^{Q}\left(t_{1}, t_{2}\right)$, given $Q$, are rewritten as $m_{X}(t ; q), C_{X X}\left(t_{1}, t_{2} ; q\right)$ and $C_{X V}\left(t_{1}, t_{2}\right.$; $q)$. Therefore, the mean function and the covariance function of stochastic process $X$, and the cross-covariance functions with the stochastic process $V$, are given by

$$
\begin{aligned}
m_{X}(t) & =\int_{\mathbb{D}_{Q}} m_{X}(t ; q) \mathrm{P}_{Q}(d q) \\
C_{X X}\left(t_{1}, t_{2}\right) & =\int_{\mathbb{D}_{Q}} C_{X X}\left(t_{1}, t_{2} ; q\right) \mathrm{P}_{Q}(d q), \\
C_{X V}\left(t_{1}, t_{2}\right) & =\int_{\mathbb{D}_{Q}} C_{X V}\left(t_{1}, t_{2} ; q\right) \mathrm{P}_{Q}(d q) .
\end{aligned}
$$

Taking into account previous definitions and notations, it is possible to state that the problem being solved in this paper consists in constructing a scheme for the calculation of the vector-valued function $m_{X}(t)$ and the matrix-valued function $C_{X X}\left(t_{1}, t_{2}\right)$ for all $t, t_{1}$ and $t_{2}$ in $\left[t_{0}, T\right]$. The scheme proposed consists of two stages: (i) calculation of the first- and second-order conditional moment functions of stochastic process $X^{Q}$ given $Q$ and then, (ii) calculation of the unconditional first- and second-order moment functions of stochastic process $X$.

\section{Equations for the first- and second-order conditional moment functions}

Applying the conditional mathematical expectation (given $Q$ ) to all terms of Eqs. (4) yields

$$
\begin{gathered}
\dot{m}_{X}^{Q}(t)=\mathcal{A}(t ; Q) m_{X}^{Q}(t)+\int_{t^{\prime}}^{t} \mathcal{B}(t, \tau ; Q) m_{X}^{Q}(\tau) d \tau+\mathcal{G}(t ; Q) v(t), \\
t_{0} \leqslant t^{\prime}<t \leqslant T, \quad m_{X}^{Q}\left(t^{\prime}\right)=m_{X}^{Q \prime}, \quad m_{X}^{Q}\left(t^{\prime}\right)=m_{X_{0}} \quad \text { for } \quad t^{\prime}=t_{0} .
\end{gathered}
$$

To derive the equation for $\mathcal{C}_{X X}^{Q}\left(t_{1}, t_{2}\right)$, it is necessary to extend the state vector of Eqs. (4) to the new random state vector with values in $\mathbb{R}^{n+m}$ defined by $Y(t)=$ $(X(t), V(t))$. Then the conditional mean function with values in $\mathbb{R}^{n+m}$ of the stochastic process $Y$ (given $Q$ ) is written as

$$
m_{Y}^{Q}(t)=\left(m_{X}^{Q}(t), 0\right)
$$

and the conditional covariance function with values in $\mathbb{R}^{(n+m) \times(n+m)}$ is such that

$$
\mathcal{C}_{Y Y}^{Q}\left(t_{1}, t_{2}\right)=\left[\begin{array}{ll}
\mathcal{C}_{X X}^{Q}\left(t_{1}, t_{2}\right) & \mathcal{C}_{X V}^{Q}\left(t_{1}, t_{2}\right) \\
\mathcal{C}_{X V}^{Q}\left(t_{2}, t_{1}\right)^{\top} & C_{V V}\left(t_{1}, t_{2}\right)
\end{array}\right]
$$


The stochastic process $Y$ indexed by $t \in\left[t_{0}, T\right]$ with values in $\mathbb{R}^{n+m}$ is governed by the following stochastic integro-differential equation,

$$
\begin{aligned}
d Y(t) & =\left\{\overline{\mathcal{A}}(t ; Q) Y(t)+\int_{t^{\prime}}^{t} \overline{\mathcal{B}}(t, \tau ; Q) Y(\tau) d \tau+\overline{\mathcal{G}}(t ; Q) v(t)\right\} d t+\bar{R} d W(t), \\
t_{0} & \leqslant t^{\prime}<t \leqslant T, \quad Y\left(t^{\prime}\right)=Y^{\prime}, \quad Y^{\prime}=\left(X_{0}, V_{0}\right) \quad \text { for } \quad t^{\prime}=t_{0} .
\end{aligned}
$$

In Eqs. (13), the matrices $\overline{\mathcal{A}}(t ; Q)$ and $\overline{\mathcal{B}}(t ; Q)$ in $\mathbb{R}^{(n+m) \times(n+m)}$, the matrix $\overline{\mathcal{G}}(t ; Q)$ in $\mathbb{R}^{(n+m) \times m}$ and the matrix $\bar{R}(t ; Q)$ in $\mathbb{R}^{(n+m) \times r}$ are defined by blocks as follows:

$$
\begin{array}{cc}
\overline{\mathcal{A}}(t ; Q)=\left[\begin{array}{cc}
\mathcal{A}(t ; Q) & \mathcal{G}(t ; Q) \\
0 & H
\end{array}\right], \quad \overline{\mathcal{B}}(t ; Q)=\left[\begin{array}{cc}
\mathcal{B}(t ; Q) & 0 \\
0 & 0
\end{array}\right], \\
\overline{\mathcal{G}}(t ; Q)=\left[\begin{array}{c}
\mathcal{G}(t ; Q) \\
0
\end{array}\right], & \bar{R}=\left[\begin{array}{l}
0 \\
R
\end{array}\right] .
\end{array}
$$

Let us introduce the centered stochastic process $Z$ indexed by $t \in\left[t_{0}, T\right]$ with values in $\mathbb{R}^{n+m}$ such that $Z(t)=Y(t)-m_{Y}^{Q}(t)$. Then the process $Z$ is the solution of the following stochastic integro-differential equation:

$$
\begin{aligned}
d Z(t)=\left\{\overline{\mathcal{A}}(t ; Q) Z(t)+\int_{t^{\prime}}^{t} \overline{\mathcal{B}}(t, \tau ; Q) Z(\tau) d \tau\right\} d t & \\
& +\bar{R} d W(t), \quad t_{0} \leqslant t^{\prime}<t \leqslant T, \quad Z\left(t^{\prime}\right)=Y^{\prime}-m_{Y}^{Q \prime} .
\end{aligned}
$$

As previously, the conditional stochastic process $Z$ as solution of Eqs. (14), for given $Q$, will be denoted by $Z^{Q}$. Since $\mathcal{C}_{Y Y}^{Q}\left(t_{1}, t_{2}\right)=\mathcal{C}_{Y Y}^{Q \top}\left(t_{2}, t_{1}\right)=\mathcal{C}_{Z Z}^{Q}\left(t_{1}, t_{2}\right)=$ $\mathbf{E}\left\{Z^{Q}\left(t_{1}\right) Z^{Q \top}\left(t_{2}\right)\right\}$, from Eq. (14), it can be deduced the two following equations, for $t_{0} \leqslant t^{\prime}<t \leqslant T$, given $\mathcal{C}_{Y Y}^{Q}\left(t^{\prime}, t^{\prime}\right)$ :

$$
\begin{gathered}
\text { (i) } \frac{\partial}{\partial t} \mathcal{C}_{Y Y}^{Q}\left(t, t^{\prime}\right)=\overline{\mathcal{A}}(t ; Q) \mathcal{C}_{Y Y}^{Q}\left(t, t^{\prime}\right)+\int_{t^{\prime}}^{t} \overline{\mathcal{B}}(t, \tau ; Q) \mathcal{C}_{Y Y}^{Q}\left(\tau, t^{\prime}\right) d \tau \\
\text { (ii) } \frac{d}{d t} \mathcal{C}_{Y Y}^{Q}(t, t)=\overline{\mathcal{A}}(t ; Q) \mathcal{C}_{Y Y}^{Q}(t, t)+\left\{\overline{\mathcal{A}}(t ; Q) \mathcal{C}_{Y Y}^{Q}(t, t)\right\}^{\top} \\
+\int_{t^{\prime}}^{t}\left[\overline{\mathcal{B}}(t, \tau ; Q) \mathcal{C}_{Y Y}^{Q}(\tau, t)+\left\{\overline{\mathcal{B}}(t, \tau ; Q) \mathcal{C}_{Y Y}^{Q}(\tau, t)\right\}^{\top}\right] d \tau+2 \pi \bar{R} \bar{R}^{\top}
\end{gathered}
$$

Eqs. (15), (16) must be solved with the following initial conditions:

$$
\mathcal{C}_{Y Y}^{Q}\left(t^{\prime}, t^{\prime}\right)=\mathcal{C}_{Y Y}^{Q}{ }^{\prime}, \quad \mathcal{C}_{Y Y}^{Q}{ }^{\prime}=\mathcal{C}_{Y Y}^{Q}\left(t_{0}, t_{0}\right)=C_{Y_{0} Y_{0}}=\left[\begin{array}{cc}
0 & 0 \\
0 & C_{V_{0} V_{0}}
\end{array}\right] \quad \text { for } t^{\prime}=t_{0} .
$$




\section{A short review on the Green matrix-valued function for a linear integro- differential equation and schemes for its approximate computation}

Let us assume that we have to solve the following deterministic IDE,

$$
\dot{z}(t)=\mathcal{A}(t) \boldsymbol{z}(t)+\int_{t^{\prime}}^{t} \mathcal{B}(t, \tau) \boldsymbol{z}(\tau) d \tau+f(t), \quad t_{0} \leqslant t^{\prime}<t,
$$

with the initial condition,

$$
z\left(t^{\prime}\right)=z^{\prime}, \quad z^{\prime}=z_{0} \quad \text { for } \quad t^{\prime}=t_{0} .
$$

The solution $z(t) \in \mathbb{R}^{n}$ of Eqs. (17) and (18) can be written as [24]

$$
z(t)=\Phi\left(t, t^{\prime}\right) z^{\prime}+\int_{t^{\prime}}^{t} \Phi(t, \tau) f(\tau) d \tau, \quad t>t^{\prime}
$$

where $\Phi\left(s, s_{0}\right)$ is the Green matrix-valued function associated with Eq. (17) and is governed by the equation,

$$
\begin{gathered}
\frac{\partial}{\partial s} \Phi\left(s, s_{0}\right)=\mathcal{A}(s) \Phi\left(s, s_{0}\right)+\int_{s_{0}}^{s} \mathcal{B}(s, \tau) \Phi\left(\tau, s_{0}\right) d \tau, \quad s>s_{0} \\
\Phi\left(s_{0}, s_{0}\right)=E .
\end{gathered}
$$

Solvers for deterministic integro-differential equations (see Section 1) include schemes requiring to calculate Green functions. But the most of them requires a great amount of analytic calculations.

It should be noted that a modern approach for building of approximate analytic solutions for problems from different scientific domains, including stochastic ones, consists in using symbolic numerical algorithms [28], which include a majority of schemes referred in the review (Section 1), but are not suitable for the problem that has to be solved. The successive approximation method [24] is one of applicable schemes. Unfortunately, the use of this scheme is hindered by the fact that a large amount of integrals has to be computed in closed form, which is not possible even by using computer algebra programs.

Nevertheless, it is feasible to represent $\Phi\left(s, s_{0}\right)$, without any integrations, in the following form [29]:

$$
\Phi\left(s, s_{0}\right)=E+\sum_{\ell=1}^{+\infty} \frac{\Phi_{\ell}\left(s_{0}, s_{0}\right)}{\ell !}\left(s-s_{0}\right)^{\ell}, \quad \Phi_{\ell}\left(s, s_{0}\right)=\frac{\partial^{\ell} \Phi\left(s, s_{0}\right)}{\partial s^{\ell}} .
$$


Using this approach, the coefficients of the Taylor series in Eq. (21) can be obtained by successive differentiations of Eq. (20) with respect to $s$. Limiting the development to the $L$-th derivative, Eq. (21) yields

$$
\widehat{\Phi}_{[L]}\left(s, s_{0}\right)=E+\sum_{\ell=1}^{L} \frac{\Phi_{\ell}\left(s_{0}, s_{0}\right)}{\ell !}\left(s-s_{0}\right)^{\ell},
$$

with an accuracy $O\left[\left(s-s_{0}\right)^{L}\right]$ (see [23]). The coefficients for $L=4$ are given in Appendix A.

In Section 5 two forms of Eq. (22) are used for exact analytical representations of the conditional vector-valued mean function and matrix-valued covariance function for all $t \geqslant t_{0}$. Further, in Section 6 these forms are adapted for approximate step-by-step analytical representations of the corresponding conditional functions in mesh nodes.

\section{Green matrix-valued functions for the first- and second-order conditional moment functions}

5.1. Formal scheme for calculation of the conditional vector-valued mean function

Comparing Eqs. (10) and (11) with Eqs. (17) and (18), and using Eq. (19), it can be deduced that the conditional vector-valued mean function $m_{X}^{Q}(t)$ is explicitly given by

$$
m_{X}^{Q}(t)=\Phi^{[m]}\left(t, t^{\prime}\right) m_{X}^{Q \prime}+\int_{t^{\prime}}^{t} \Phi^{[m]}(t, \tau) \mathcal{G}(\tau ; Q) v(\tau) d \tau,
$$

where the Green matrix-valued function $\Phi\left(s, s_{0}\right)$ associated with $m_{X}^{Q}(t)$ is rewritten as $\Phi^{[m]}\left(s, s_{0}\right)$ and is calculated using Eqs. (20) and (21) with

$$
\mathcal{A}(t)=\mathcal{A}(t ; Q), \quad \mathcal{B}(t, \tau)=\mathcal{B}(t, \tau ; Q) .
$$

5.2. Formal scheme for calculation of the conditional matrix-valued covariance function

Let us start with Eq. (15) satisfied by the conditional matrix-valued covariance function $\mathcal{C}_{Y Y}^{Q}\left(t, t^{\prime}\right)$ for $t>t^{\prime}$. We can state that the Green matrix-valued function $\Phi\left(s, s_{0}\right)$ governed by Eq. (20) and associated with $\mathcal{C}_{Y Y}^{Q}\left(t, t^{\prime}\right)$, can be rewritten as $\Phi^{[C]}\left(t, t^{\prime}\right)$ and is calculated using Eqs. (20) and (21) with

$$
\mathcal{A}(t)=\overline{\mathcal{A}}(t ; Q), \quad \mathcal{B}(t)=\overline{\mathcal{B}}(t ; Q) .
$$


From Eq. (15), it can be deduced that

$$
\mathcal{C}_{Y Y}^{Q}\left(t, t^{\prime}\right)=\Phi^{[C]}\left(t, t^{\prime}\right) \mathcal{C}_{Y Y}^{Q}\left(t^{\prime}, t^{\prime}\right), \quad t_{0} \leqslant t^{\prime}<t \leqslant T .
$$

Now let's turn to the calculation of the conditional matrix-valued covariance function $\mathcal{C}_{Y Y}^{Q}(t, t)$ satisfying Eq. (16) for $t_{0} \leqslant t^{\prime}<t \leqslant T$. Notice that the conditional vector-valued stochastic process $Z^{Q}$ can be represented by using $\Phi^{[C]}(\cdot, \cdot)$ as the solution of Eq. (14) (for given $Q$ ) in the following form:

$$
Z^{Q}(t)=\Phi^{[C]}(t, s) Z^{Q}\left(t^{\prime}\right)+\int_{t^{\prime}}^{t} \Phi^{[C]}(t, \tau) \bar{R} d W(\tau), \quad t^{\prime}<t .
$$

This form allows the conditional matrix-valued covariance function $\mathcal{C}_{Y Y}^{Q}(t, t)=$ $\mathbf{E}\left\{Z^{Q}(t) Z^{Q \top}(t)\right\}$, for all $t_{0} \leqslant t^{\prime}<t \leqslant T$, to be calculated by

$$
\begin{aligned}
\mathcal{C}_{Y Y}^{Q}(t, t)=\Phi^{[C]}\left(t, t^{\prime}\right) \mathcal{C}_{Y Y}^{Q}\left(t^{\prime}, t^{\prime}\right) & \Phi^{[C]}\left(t, t^{\prime}\right) \\
& +2 \pi \int_{t^{\prime}}^{t} \Phi^{[C]}(t, \tau) \bar{R}\left\{\Phi^{[C]}(t, \tau) \bar{R}\right\}^{\top} d \tau .
\end{aligned}
$$

\section{Approximations for the first- and second-order conditional moment func- tions}

The following time grid is introduced,

$$
\begin{gathered}
t_{0}=\tau_{0}<\tau_{1}<\ldots<\tau_{k-1}<\tau_{k}<\ldots<\tau_{N}=T \\
h_{k}=\tau_{k}-\tau_{k-1} \quad \text { for } \quad k=1,2, \ldots, N \quad \text { with } \quad \max _{k} h_{k}=h_{*} \ll 1 .
\end{gathered}
$$

In this section, the first- and second-order conditional moment functions $m_{X, k}^{Q}=$ $m_{X}^{Q}\left(\tau_{k}\right)$ and $\mathcal{C}_{Y Y, k \nu}^{Q}=\mathcal{C}_{Y Y}^{Q}\left(\tau_{k}, \tau_{\nu}\right)$ are computed at the grid nodes for $k$ and $\nu$ in $\{1, \ldots, N\}$ with the accuracy $O\left(h_{k}^{L}\right)$.

\subsection{Approximate scheme for calculation of the first-order conditional moment function}

The numerical approximation of the conditional vector-valued mean function $m_{X}^{Q}(t)$, such that

$$
m_{X}^{Q}(t)=\Phi^{[m]}\left(t, t^{\prime}\right) m_{X}^{Q \prime}+\int_{t^{\prime}}^{t} \Phi^{[m]}(t, \tau) \mathcal{G}(\tau ; Q) v(\tau) d \tau,
$$

for $t^{\prime}=\tau_{k-1}, t=\tau_{k}$, can be written as

$$
\widehat{m}_{X, k}^{Q}=\widehat{\Phi}_{[L]}^{[m]}\left(\tau_{k}, \tau_{k-1}\right) \widehat{m}_{X, k-1}^{Q}+\int_{\tau_{k-1}}^{\tau_{k}} \widehat{\Phi}_{[L]}^{[m]}\left(\tau_{k}, \tau\right) \mathcal{G}(\tau ; Q) v(\tau) d \tau,
$$




$$
k=1,2, \ldots, N, \quad \widehat{m}_{X, 0}^{Q}=m_{X_{0}},
$$

where $\widehat{\Phi}_{[L]}^{[m]}\left(\tau_{k}, \tau\right)=\widehat{\Phi}_{[L]}\left(\tau_{k}, \tau\right)$ with taking into account Eqs. (24). The integral in Eq. (27) can be computed by using, for instance, of the Simpson rule.

\subsection{Approximate scheme for calculation of the second-order conditional moment function}

Similarly, the conditional matrix-valued covariance function that verifies the equations, for $t_{0} \leqslant t^{\prime}<t \leqslant T$,

$$
\mathcal{C}_{Y Y}^{Q}\left(t, t^{\prime}\right)=\Phi^{[C]}\left(t, t^{\prime}\right) \mathcal{C}_{Y Y}^{Q}\left(t^{\prime}, t^{\prime}\right)
$$

can numerically be approximated, for $k=0,1, \ldots, N-1$ and $\nu=k+1, \ldots, N$, by

$$
\widehat{\mathcal{C}}_{Y Y, \nu k}^{Q}=\widehat{\Phi}^{[C]}\left(\tau_{\nu}, \tau_{\nu-1}\right) \widehat{\mathcal{C}}_{Y Y, \nu-1, k}^{Q}
$$

in which $\widehat{\Phi}_{[L]}^{[C]}\left(\tau_{\nu}, \tau_{\nu-1}\right)=\widehat{\Phi}_{[L]}\left(\tau_{\nu}, \tau_{\nu-1}\right)$ with taking into account Eqs. (25).

Finally, for $t_{0} \leqslant t^{\prime}<t \leqslant T$, the conditional matrix-valued covariance function $\mathcal{C}_{Y Y}^{Q}(t, t)$, that verifies the Eq. (26), can numerically be approximated, for $k=1, \ldots, N$, by

$$
\begin{aligned}
\widehat{\mathcal{C}}_{Y Y, k k}^{Q}=\widehat{\Phi}_{[L]}^{[C]}\left(\tau_{k}, \tau_{k-1}\right) \widehat{\mathcal{C}}_{Y Y, k-1, k-1}^{Q} \widehat{\Phi}_{[L]}^{[C] \top}\left(\tau_{k}, \tau_{k-1}\right) & \\
& +2 \pi \int_{\tau_{k-1}}^{\tau_{k}} \Phi_{L}^{[C]}\left(\tau_{k}, \tau\right) \bar{R}\left\{\Phi_{L}^{[C]}\left(\tau_{k}, \tau\right) \bar{R}\right\}^{\top} d \tau, \quad \widehat{\mathcal{C}}_{Y Y, 00}^{Q}=\mathcal{C}_{Y_{0} Y_{0}}
\end{aligned}
$$

\section{Stochastic solver}

In the previous sections, the analytic procedure has been proposed for constructing approximations of the first- and second-order conditional moment functions $m_{X}^{Q}(t)=m_{X}(t ; q)$ and $\mathcal{C}_{X X}^{Q}\left(t_{1}, t_{2}\right)=C_{X X}\left(t_{1}, t_{2} ; q\right)$ of stochastic state vector $X$, for given $Q=q$. In order to obtain the first- and second-order unconditional moment functions $m_{X}(t)$ and $C_{X X}\left(t_{1}, t_{2}\right)$ (the solution of the problem was formulated in Section 2), it is necessary to complete the procedure using Eqs. (7), (9a) and (9b). Clearly, this procedure can only be a numerical one because symbolic integration for the probability distribution $\mathrm{P}_{Q}(d q)$ of random vector $Q$, which is assumed to be arbitrary, cannot be carried out. Therefore, the Monte Carlo numerical method is used for computing the integrals in Eqs. (7), (9a), and (9b). 
Let $M$ be the number of independent realizations, $q_{1}, \ldots, q_{M}$, of random vector $Q$ whose probability distribution is $\mathrm{P}_{Q}(d q)$. The Monte Carlo approximations can be written as

$$
\widehat{\mathrm{P}}_{X}(d \chi ; t)=\frac{1}{M} \sum_{\ell=1}^{M} \mathrm{P}_{X Q}\left(d \chi ; t \mid q_{\ell}\right)
$$

and, for all $k$ and $\nu$ in $\{1, \ldots, N\}$, as

$$
\widehat{m}_{X}\left(\tau_{k}\right)=\frac{1}{M} \sum_{\ell=1}^{M} m_{X}\left(\tau_{k} ; q_{\ell}\right), \quad \widehat{C}_{X X}\left(\tau_{k}, \tau_{\nu}\right)=\frac{1}{M} \sum_{\ell=1}^{M} C_{X X}\left(\tau_{k}, \tau_{\nu} ; q_{\ell}\right),
$$

for which the accuracy is $O\left(M^{-1 / 2}\right)$. Moreover, using the estimators of mathematical expectations and variances for $X_{i}\left(\tau_{k}\right)$, for $i$ in $\{1, \ldots, n\}$ and $k$ in $\{1, \ldots, N\}$, we can find approximations of their probability density functions (PDFs) as

$$
\widehat{p}_{X_{i}}\left(x_{i}, \tau_{k}\right)=\frac{1}{M} \sum_{\ell=1}^{M} \mathcal{N}\left(x_{i} ; \widehat{m}_{X_{i}}\left(\tau_{k} ; q_{\ell}\right), \widehat{C}_{X_{i} X_{i}}\left(\tau_{k}, \tau_{k} ; q_{\ell}\right)\right)
$$

where

$$
\mathcal{N}\left(x ; a, \sigma^{2}\right)=\frac{1}{\sqrt{2 \pi} \sigma} \exp \left[-\frac{(x-a)^{2}}{2 \sigma^{2}}\right]
$$

\section{Application to a dynamical system with a thermoviscoelastic medium}

\subsection{Definition of the dynamical system}

We consider the mechanical system defined in Fig. 1, constituted of two rigid bodies $\Omega_{1}$ and $\Omega_{2}$ with mass $M_{1}>0$ and $M_{2}>0$ respectively. Each body has only one degree of freedom corresponding to its displacement in the same given direction. Body $\Omega_{1}$ is linked to body $\Omega_{2}$ and to the moving base. The link between $\Omega_{1}$ and $\Omega_{2}$ is made up of a linear elastic dissipative mechanical component which is modeled by a linear spring with constant stiffness $K>0$ and by a linear dashpot of viscous type with constant damping coefficient $D>0$. The link between $\Omega_{1}$ and the moving base is made up of a thermoviscoelastic mechanical component (that is characterized by two functions of time, $k_{0}(t, t)$ and $k(t, \tau)$, which will be defined later with the constitutive equation).

An absolute reference frame is introduced for which the unit vector basis (corresponding to the considered displacement) is denoted by $\boldsymbol{J}_{0}$. The base is moving in the direction $\boldsymbol{J}_{0}$. A relative reference frame linked to the moving base is also 
introduced for which the unit vector basis (corresponding to the considered displacement) is denoted by $\boldsymbol{j}$.

The linear dynamics of this mechanical system is analyzed around a reference configuration which corresponds to a static equilibrium state for which the mechanical components are assumed to be without prestresses. The initial time for the time-evolution problem is chosen as $t_{0}=0$. In the absolute reference frame and at time $t$, the displacement of the moving base is written as $U_{s}(t) \boldsymbol{J}$. In the relative reference frame linked to the moving base and at time $t$, the displacement of body $\Omega_{1}$ (or $\Omega_{2}$ ) is written as $U_{1}(t) \boldsymbol{j}$ (or $U_{2}(t) \boldsymbol{j}$ ).

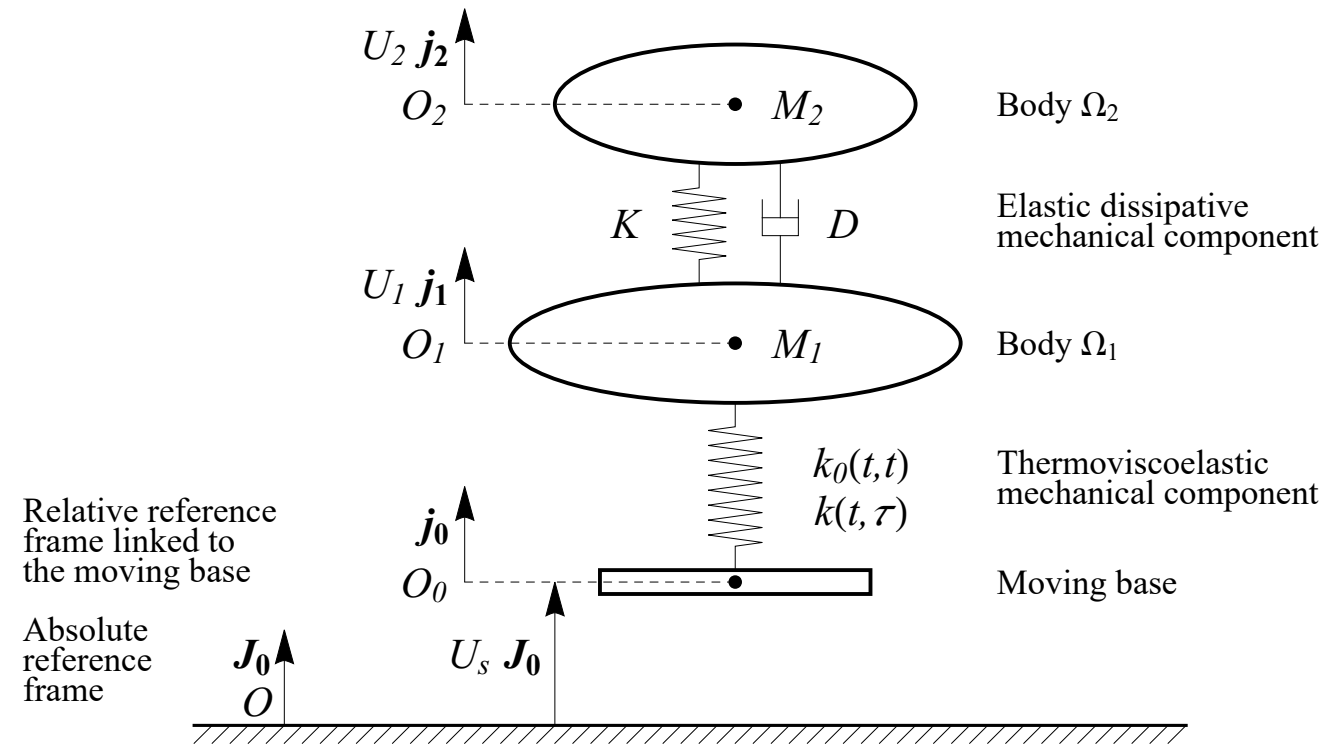

Figure 1: Scheme defining the mechanical system.

For defining the constitutive equation of the thermoviscoelastic mechanical component, the bottom end of this component is assumed to be fixed and the top end of the component is free and submitted, at time $t$, to a force $f(t) \boldsymbol{j}$ inducing a displacement $U_{1}(t) \boldsymbol{j}$. Using the non-isothermal linear viscoelasticity theory (see, for instance, $[30,31,8])$, the constitutive equation can then be written as

$$
f(t)=k_{0}(t, t ; Q) U_{1}(t)+\int_{0}^{t} k(t, \tau ; Q) U_{1}(\tau) d \tau, \quad 0<t \leqslant T,
$$

in which $(t, \tau) \mapsto k_{0}(t, \tau ; Q)$ is a positive-valued function that is defined on $0<$ $\tau \leqslant t \leqslant T$ and is assumed to be infinitely differentiable in $(t, \tau)$. Further, $(t, \tau) \mapsto$ $k(t, \tau ; Q)=\partial k_{0}(t, \tau ; Q) / \partial \tau$ is a negative-valued function which is defined on $0<\tau \leqslant t \leqslant T$ and which is then integrable in $\tau$ on $] 0, t]$ for all $t$ in $] 0, T]$. Uncertain vector-valued parameter $Q$ and functions $k_{0}$ and $k$ will precisely be defined later. 


\subsection{Equations for the dynamical system}

For $j=1,2$, let $\dot{U}_{j}(t)=d U_{j}(t) / d t$ be the velocity and let $\ddot{U}_{j}(t)=d^{2} U_{j}(t) / d t^{2}$ be the acceleration. The dynamics is studied around a reference configuration that corresponds to a static equilibrium at rest without prestresses. At initial time, the displacements and the velocities are thus zero. The dynamic equilibrium equation around the reference configuration is written, for all $t$ in $] 0, T]$, as

$$
\begin{gathered}
M_{1}\left(\ddot{U}_{1}(t)+\ddot{U}_{s}(t)\right)=-K\left(U_{1}(t)-U_{2}(t)\right)-D\left(\dot{U}_{1}(t)-\dot{U}_{2}(t)\right) \\
-k_{0}(t, t ; Q) U_{1}(t)-\int_{0}^{t} k(t, \tau ; Q) U_{1}(\tau) d \tau, \\
M_{2}\left(\ddot{U}_{2}(t)+\ddot{U}_{s}(t)\right)=-K\left(U_{2}(t)-U_{1}(t)\right)-D\left(\dot{U}_{2}(t)-\dot{U}_{1}(t)\right),
\end{gathered}
$$

with the initial conditions,

$$
U_{1}(0)=\dot{U}_{1}(0)=U_{2}(0)=\dot{U}_{2}(0)=0 .
$$

Let $\omega_{1}, \omega_{2}, \xi$ and $\mu$ be the positive real parameters such that

$$
\omega_{1}=\sqrt{K / M_{1}}, \quad \omega_{2}=\sqrt{K / M_{2}}, \quad \xi=D /\left(2 M_{2} \omega_{2}\right), \quad \mu=M_{2} / M_{1} .
$$

Further, let $(t, \tau) \mapsto \widetilde{k}_{0}(t, \tau ; Q)$ and $(t, \tau) \mapsto \widetilde{k}(t, \tau ; Q)$ be the functions such that

$$
\widetilde{k}_{0}(t, \tau ; Q)=k_{0}(t, \tau, Q) / M_{1}, \quad \widetilde{k}(t, \tau ; Q)=k(t, \tau ; Q) / M_{1} .
$$

Then Eqs. (30a) and (30b) can be rewritten as

$$
\begin{gathered}
\ddot{U}_{1}(t)=-2 \xi \sqrt{\mu} \omega_{1}\left(\dot{U}_{1}(t)-\dot{U}_{2}(t)\right)-\omega_{1}^{2}\left(U_{1}(t)-U_{2}(t)\right) \\
-\widetilde{k}_{0}(t, t ; Q) U_{1}(t)-\int_{0}^{t} \widetilde{k}(t, \tau ; Q) U_{1}(\tau) d \tau-\ddot{U}_{s}(t), \\
\ddot{U}_{2}(t)=-2 \xi \omega_{2}\left(\dot{U}_{2}(t)-\dot{U}_{1}(t)\right)-\omega_{2}^{2}\left(U_{2}(t)-U_{1}(t)\right)-\ddot{U}_{s}(t) .
\end{gathered}
$$

\subsection{Stochastic modeling of the moving base acceleration}

The acceleration $\ddot{U}_{s}$ of the moving base is modeled by a second-order, Gaussian, nonstationary, centered stochastic process $\left\{\ddot{U}_{s}(t), t \in[0, T]\right\}$ which is such that $\ddot{U}_{s}(t)=g(t) V(t)$. The real-valued function $g$ is defined on $[0, T]$ and is such that $g(t)=\left(1+\left(t / t_{g}\right)^{a_{g}}\right)^{-1}, t \in[0, T]$, in which $0<t_{g}<T$ and $a_{g}>0$. Note that such the form is a usual model for simple nonstationary random fluctuations in stochastic mechanics, for example, in simulation of earthquakes.

The stochastic process $\{V(t), t \in \mathbb{R}\}$ is a second-order, Gaussian, stationary, centered, mean-square continuous stochastic process indexed by $\mathbb{R}$ with values in 
$\mathbb{R}$ for which the autocovariation function $C_{V}(\tau)=\mathbf{E}\{V(t+\tau) V(t)\}$ is written as

$$
C_{V}(\tau)=\int_{\mathbb{R}} e^{i \omega t} S_{V}(\omega) d \omega, \quad S_{V}(\omega)=\frac{a_{V}^{2}}{b_{V}^{2}+\omega^{2}}, \quad a_{V}>0, \quad b_{V}>0,
$$

where $S_{V}(\omega)$ is the power spectral density function. For all fixed $t$ in $\mathbb{R}$, let $\sigma_{V}$ be the standard deviation of the random variable $V(t)$ which is such that

$$
\sigma_{V}^{2}=C_{V}(0)=\int_{\mathbb{R}} S_{V}(\omega) d \omega .
$$

Consequently, the stochastic process $\{V(t), t \in[0, T]\}$ can be generated as the (stationary) solution of the following Itô stochastic differential equation with random initial condition,

$$
\left.\left.d V(t)=-b_{V} V(t) d t+a_{V} d W(t), \quad t \in\right] 0, T\right], \quad V(0)=V_{0} \quad \text { a.s. }
$$

in which $W$ is the Wiener stochastic process introduced in Section 2 with $m=1$ and where the probability distribution of the random variable $V_{0}$ is the invariant measure $\mathrm{P}_{V}$ which is the Gaussian probability measure with zero mean and standard deviation equal to $\sigma_{V}$.

\subsection{Stochastic integro-differential equations for the dynamical system}

Taking $n=4, m=1, t_{0}=0$ and $v(t)=0$, it can be viewed that Eqs. (33a), (33b), and (31) can then be written as Eq. (4) with

$$
\begin{array}{ccc}
\mathcal{A}(t ; Q)=\left[\begin{array}{cccc}
-\xi_{1} & \xi_{1} & -\bar{\omega}_{1}^{2} & \omega_{1}^{2} \\
\xi_{2} & -\xi_{2} & \omega_{2}^{2} & -\omega_{2}^{2} \\
1 & 0 & 0 & 0 \\
0 & 1 & 0 & 0
\end{array}\right], \quad \mathcal{B}(t, \tau ; Q)=\left[\begin{array}{cccc}
0 & 0 & \bar{k} & 0 \\
0 & 0 & 0 & 0 \\
0 & 0 & 0 & 0 \\
0 & 0 & 0 & 0
\end{array}\right] \\
\mathcal{G}(t ; Q)=-\left[\begin{array}{c}
g(t) \\
g(t) \\
0 \\
0
\end{array}\right], & X(t)=\left[\begin{array}{l}
\dot{U}_{1}(t) \\
\dot{U}_{2}(t) \\
U_{1}(t) \\
U_{2}(t)
\end{array}\right], & X_{0}=\left[\begin{array}{l}
0 \\
0 \\
0 \\
0
\end{array}\right]
\end{array}
$$

where $\xi_{1}=2 \xi \sqrt{\mu} \omega_{1}, \xi_{2}=2 \xi \omega_{2}, \bar{\omega}_{1}^{2}=\omega_{1}^{2}+\widetilde{k}_{0}(t, t ; Q), \bar{k}=-\widetilde{k}(t, \tau ; Q)$. In addition, Eqs. (34) are written as Eqs. (5) with $m=1, t_{0}=0, H=\left[-b_{V}\right]$, $R=\left[a_{V}\right]$. 
8.5. Kernel of the constitutive equation for the thermoviscoelastic mechanical component

In this section, we define the kernel $\widetilde{k}_{0}(t, \tau ; Q)$ introduced in Section 8.1, which is presently derived from the model proposed in [32]. Random vector $Q$ of the uncertain parameters will be defined in Section 8.6. Let $\theta_{t}$ be the temperature of the mechanical component defined on $[0, T]$ and written as $\theta_{t}=a_{\theta}+b_{\theta} t / T$, $a_{\theta}>0, b_{\theta}>0$.

The kernel $\widetilde{k}_{0}(t, \tau ; Q)$ is then defined, for $0<\tau \leqslant t \leqslant T$, by

$$
\widetilde{k}_{0}(t, \tau ; Q)=K_{0}\left(\theta_{t}\right)\left[1-\sum_{\ell=1}^{2} \mu_{\ell}\left(\theta_{t}\right)\left(1-\exp \left\{-\frac{\tau}{T_{\ell}\left(\theta_{t}\right)}\right\}\right)\right] .
$$

For all $t$ in $[0, T]$, the initial elastic property, $K_{0}\left(\theta_{t}\right)>0$, which depends on the temperature, is written as $K_{0}\left(\theta_{t}\right)=a_{K_{0}} /\left(\left(b_{K_{0}} \theta_{t}-1\right)\right.$ with $a_{K_{0}}>0, b_{K_{0}}>0$, where the constant $b_{K_{0}}$ is such that $b_{K_{0}} \theta_{t}-1>0$.

In the present application, $T_{1}\left(\theta_{t}\right)$ will be the large relaxation time. This time and the associated dimensionless parameter $\mu_{1}\left(\theta_{t}\right)$ are assumed to be independent of $\theta_{t}$ and are written as $T_{1}\left(\theta_{t}\right)=a_{T_{1}}, \mu_{1}\left(\theta_{t}\right)=a_{\mu_{1}}$ with $a_{T_{1}}>0, a_{\mu_{1}}>0$. The relaxation time $T_{2}\left(\theta_{t}\right)$ will be the small relaxation time. This time and the associated dimensionless parameter $\mu_{2}\left(\theta_{t}\right)$ are assumed to be dependent on $\theta_{t}$ and are written as

$$
\begin{gathered}
T_{2}\left(\theta_{t}\right)=\frac{a_{T_{2}}}{b_{T_{2}} \theta_{t}+\left(c_{T_{2}} / \theta_{t}\right)^{d_{T_{2}}}} \text { with } a_{T_{2}}>0, \quad b_{T_{2}}>0, \quad c_{T_{2}}>0, \quad d_{T_{2}}>0, \\
\mu_{2}\left(\theta_{t}\right)=a_{\mu_{2}}-b_{\mu_{2}} \theta_{t} \quad \text { with } \quad a_{\mu_{2}}>0, \quad b_{\mu_{2}}>0,
\end{gathered}
$$

where the constants $a_{\mu_{2}}$ and $b_{\mu_{2}}$ are such that $a_{\mu_{2}}-b_{\mu_{2}} \theta_{t}>0$. From Section 8.1 and from Eqs. (32) and (35), it can be deduced that

$$
\widetilde{k}(t, \tau ; Q)=-K_{0}\left(\theta_{t}\right) \sum_{\ell=1}^{2} \frac{\mu_{\ell}\left(\theta_{t}\right)}{T_{\ell}\left(\theta_{t}\right)} \exp \left\{-\frac{\tau}{T_{\ell}\left(\theta_{t}\right)}\right\} .
$$

8.6. Numerical data and definition of the probability model for the uncertain parameters

The large relaxation time $T_{1}\left(\theta_{t}\right)$, the associated dimensionless parameter $\mu_{1}\left(\theta_{t}\right)$, and parameter $d_{T_{2}}$ of the law of the small relaxation time $T_{2}\left(\theta_{t}\right)$ will be the uncertain parameters. These three random quantities are assumed to be independent.

For the numerical application, data and the probability models of the uncertain parameters are defined as follows.

1. Time duration: $T=1,200 s$. 
2. Mechanical properties: $\omega_{1}=2 \pi \mathrm{rad} / \mathrm{s}, \omega_{2}=6 \pi \mathrm{rad} / \mathrm{s}, \mu=1 / 9, \xi=0.01$.

3. Moving base acceleration: $t_{g}=300 \mathrm{~s}, a_{g}=3, a_{V}=19 \mathrm{~m} / \mathrm{s}^{5 / 2}, b_{V}=$ $19 s^{-1}$.

4. Time evolution of the temperature: $a_{\theta}=40^{\circ} \mathrm{C}, b_{\theta}=110^{\circ} \mathrm{C}$.

5. Parameters for the thermoviscoelastic mechanical component:

(a) Initial elastic property: Parameter $a_{K_{0}}$ is uncertain and is modeled by the random variable $A_{K_{0}}=\underline{a}_{K_{0}}\left(1+\delta_{K_{0}} Q_{1}\right)$ in which $Q_{1}$ is a uniform random variable on $[-1,1]$. The mean value of $A_{K_{0}}$ is $\underline{a}_{K_{0}}=$ $500 \mathrm{~N} \times \mathrm{Kg}^{-1} \times \mathrm{m}^{-1}$ and its coefficient of variation is $\delta_{K_{0}}$ which belongs to the interval $[0,0.9]$. Additionally, $b_{K_{0}}=0.04{ }^{\circ} C^{-1}$.

(b) Large relaxation time $T_{1}\left(\theta_{t}\right)$ : Parameter $a_{T_{1}}$ is uncertain and is modeled by the random variable $A_{T_{1}}=\underline{a}_{T_{1}}\left(1+\delta_{T_{1}} Q_{2}\right)$ in which $Q_{2}$ is a uniform random variable on $[-1,1]$. The mean value of $A_{T_{1}}$ is $\underline{a}_{T_{1}}=320 \mathrm{~s}$ and its coefficient of variation is $\delta_{T_{1}}$ which belongs to the interval $[0,0.9]$.

(c) Dimensionless parameter $\mu_{1}\left(\theta_{t}\right)$ : Parameter $a_{\mu_{1}}$ is uncertain and is modeled by the random variable $A_{\mu_{1}}=\underline{a}_{\mu_{1}}\left(1+\delta_{\mu_{1}} Q_{3}\right)$ in which $Q_{3}$ is a uniform random variable on $[-1,1]$. The mean value of $A_{\mu_{1}}$ is $\underline{a}_{\mu_{1}}=0.08$ and its coefficient of variation is $\delta_{\mu_{1}}$ which belongs to the interval $[0,0.9]$.

(d) Small relaxation time $T_{2}\left(\theta_{t}\right)$ : The deterministic parameters are $a_{T_{2}}=$ $60 \mathrm{~s}, b_{T_{2}}=3{ }^{\circ} \mathrm{C}^{-1}, c_{T_{2}}=73^{\circ} \mathrm{C}$. Parameter $d_{T_{2}}$ is uncertain and is modeled by the random variable $D_{T_{2}}=\underline{d}_{T_{2}}\left(1+\delta_{T_{2}} Q_{4}\right)$ in which $Q_{4}$ is a uniform random variable on $[-1,1]$ and where $\underline{d}_{T_{2}}$ is the mean value of $D_{T_{2}}$, such that $\underline{d}_{T_{2}}=11$. Its coefficient of variation is $\delta_{D_{T_{2}}}$ which belongs to the interval $[0,0.9]$.

(e) Dimensionless parameter $\mu_{2}\left(\theta_{t}\right): a_{\mu_{2}}=0.63, b_{\mu_{2}}=0.0013{ }^{\circ} C^{-1}$.

With respect to the Lebesgue measure $d q=d q_{1} d q_{2} d q_{3} d q_{4}$ on $\mathbb{R}^{4}$, the probability distribution of the random vector $Q=\left(Q_{1}, Q_{2}, Q_{3}, Q_{4}\right)$ is thus defined by the probability density function $p_{Q}(q)=p_{1}\left(q_{1}\right) \times p_{2}\left(q_{2}\right) \times p_{3}\left(q_{3}\right) \times p_{4}\left(q_{4}\right)$, in which, for $\ell=1,2,3,4, p_{\ell}\left(q_{\ell}\right)=\frac{1}{2} \mathbb{1}_{[-1,1]}\left(q_{\ell}\right)$.

The objective of the paper is not to introduce a complex probabilistic model of the parameters but to show how the methodology proposed is efficient when the parameters are random. Since the stochastic solver is the Monte Carlo method for the integration with respect to the random parameters, the methodology proposed 
is independent of the probability model chosen for the parameters. The probabilistic model that has been used for the computation has been chosen voluntary simple and could certainly be replaced by a more sophisticated informative probability model using the Maximum Entropy Principle from Information Theory, if additional information was available. This probabilistic model has been constructed using the following hypotheses: the only information that is supposed to be available consists in giving the support of each normalized random components of the vector-valued parameter. Using the Maximum Entropy principle under the constraints defined by this available information yields the probability model proposed in the paper that is to say, each random component is uniform and all these random components are mutually statistically independent (see for instance [5]).

\subsection{Numerical results and discussion}

In this subsection, the technique of implementation of the Green function method (GFM) for analyzing Eqs. (33a), (33b), and (31), is described, and the results obtained are discussed. Some details concerning the usage of GFM are explained in Section 8.7.2. The results obtained by the new method are compared with a solution constructed by the Monte Carlo method (MCM) that is regarded as a reference solution. Some details of the MCM are given in Section 8.7.1. Note that the vector mean function of $X(t)$ is equal to zero for all $t \geqslant 0$ due to the problem statement. Therefore, this function have not been calculated. Additional remarks about values of computation parameters are presented in Section 8.7.3. Different figures of time histories for estimators of $C_{Y Y}(t, t)$ components are shown and discussed in Section 8.7.4.

\subsubsection{Details of numerical calculations in the framework of pure Monte Carlo simulations}

In this section, the numerical calculations are produced in the environment of Microsoft Visual Studio with the help of Intel Fortran program designed.

For direct simulation of the system under consideration, the following simple semi-implicit modification of the Euler-Maruyama algorithm (or $\theta$-method for $\theta=0.5)$ is used:

$$
\begin{aligned}
\widehat{y}_{\ell, k+1}=\widehat{y}_{\ell k}+ & \frac{h_{0}}{2}\left[\bar{A}_{\ell k} \widehat{y}_{\ell k}+\bar{A}_{\ell, k+1} \widehat{y}_{\ell, k+1}+\right. \\
& \left.h_{0}\left(\bar{B}_{\ell, k+1, k} \widehat{y}_{\ell k}+\bar{B}_{\ell, k+1, k+1} \widehat{y}_{\ell, k+1}\right)\right]+\bar{O}{\widehat{\Delta w_{\ell k}}}, \\
& k=0,1,2, \ldots, N-1, \quad \ell=1,2, \ldots, M,
\end{aligned}
$$

where $h_{0}=T / N$ is a constant step, $\tau_{k}=k h_{0}, k \in\{0,1,2, \ldots, N\}, \widehat{y}_{\ell k}=y^{[\ell]}\left(\tau_{k}\right)$, $\left.\bar{A}_{\ell k}=\bar{A}\left(\tau_{k} ; q_{\ell}\right), \bar{B}_{\ell k j}=\bar{B}\left(\tau_{k}, \tau_{j} ; q_{\ell}\right)\right], \Delta y_{\ell k}=\widehat{y}^{[\ell]}\left(\tau_{k+1}\right)-\widehat{y}^{[\ell]}\left(\tau_{k}\right), \ell$ is the 
number of simulation. The scheme defined in Eq. (36) requires a resolution of these linear equalities for time histories $\widehat{y}_{\ell, k+1}$. The necessary matrix inversion is carried out using the Gauss elimination method.

The implementation of MCM requires a large amount of random or pseudorandom numbers with different distributions. The generation of samples for $Q$ is based on Intel Fortran build-in pseudo-random generator for uniform $(0,1)$ distributed numbers. These samples are recounted into necessary uniform $(-1,1)$ distributed numbers by an affine transformation.

To model pseudo-random standard normal $\mathcal{N}(\cdot ; 0,1)$ distributed numbers, the Marsaglia-Bray algorithm is used. After converting to samples with given standard deviations, these numbers are used as initial values of $V\left(t_{0}\right)$ and increments of $W(t)$. However, as in many another practical cases, these generators produce samples possessing statistical estimates of the mean values, variances, and covariances, which deviate from theoretical ones for a reasonable number of samples. Therefore, a normalization has been performed for all the pseudo-Gaussian samples used for the initial values and for every step of the simulation, separately. After such the normalization, the converted samples are multiplied by $\sigma_{V}$ or $\sqrt{2 \pi h_{0}}$ due to needs.

Another procedure has been used for the pseudo-uniform samples on $[-1,1]$. The matter is that it is necessary to use samples with zero estimations of the mean vector and diagonal estimations of the covariance matrix with the value $1 / 3$ for all the diagonal entries. Let $\widehat{m}_{Q}$ and $\widehat{C}_{Q Q}$ be the usual estimations of the mean vector and the covariance matrix of $Q$, which are computed with the pseudo-uniform samples, $q^{[\ell]}$, of $Q$. Then the classical equation used for the normalization, is as follows:

$$
\widehat{q}^{[\ell]}=\frac{1}{\sqrt{3}} S^{-1}\left(q^{[\ell]}-\widehat{m}_{Q}\right), \quad \ell=1,2, \ldots, M,
$$

where $S$ is the lower-triangular matrix, calculated by the Cholesky decomposition such that $\widehat{C}_{Q Q}=S S^{\top}$.

The step-by-step estimators for the vector-valued mean function and for the matrix-valued covariance function of the state vector-valued stochastic process $Y$ are computed, for $k, r$ in $\{0,1, \ldots, N\}$ and for $i$ and $j$ in $\{1,2,3,4\}$, as follows:

$$
\widehat{m}_{Y_{i}, k}=\frac{1}{M} \sum_{\ell=1}^{M} \widehat{y}_{\ell i k}, \quad \widehat{c}_{Y_{i} Y_{j}, k r}=\frac{1}{M} \sum_{\ell=1}^{M}\left(\widehat{y}_{\ell i k}-\widehat{m}_{Y_{i}, k}\right)\left(\widehat{y}_{\ell j r}-\widehat{m}_{Y_{j}, r}\right),
$$

where $c_{Y_{i} Y_{j}, k r}=c_{Y_{i} Y_{j}}\left(t_{k}, t_{r}\right)$, for $i$ and $j$ in $\{1,2,3,4\}$, are components of the matrix $C_{Y Y}\left(t_{k}, t_{r}\right)$. 
8.7.2. Details of symbolic and numerical calculations in the framework of the Green function scheme

An implementation of GFM consists of two stages. On the first stage, all analytic calculations have been produced to obtain symbolic expressions of $\Phi_{\ell}\left(s_{0}, s_{0}\right)$ for $\ell=\{1,2,3,4\}$, suitable for further numerical calculations. These necessary symbolic computations, including differentiations, substitutions, reductions of similar terms, and so on, have been made with the use of the Mathematica package. The most complex symbolic substage has been related to the differentiations of functions $\widetilde{k}_{0}(t, \tau ; Q)$ and $\widetilde{k}(t, \tau ; Q)$ because of their complicated structures. This substage was required for an achievement of effectiveness for Fortran computations. Note that a computer automatic optimized generation of corresponding Fortran program fragments by a package Maple was worse than the technique, which was mentioned above.

On the second stage, numerical calculations have been produced in the same environment of Microsoft Visual Studio with the help of other designed Intel Fortran program. In this program, as in the first one, we have formed constant parts of matrices before simulations and added variable parts to the last during simulations. The aim was to reduce computer time for both MCM and GFM. Obtaining samples for $Q$, their transformations, and statistical data processing have been done by schemes similar to those that have been represented in the previous section.

\subsubsection{Data used in calculations}

For the system under investigation, the main computation parameters has been presented in the previous sections. Here we point only four sets of values for $\delta_{K_{0}}$, $\delta_{T_{1}}, \delta_{\mu_{1}}$ and $\delta_{T_{2}}$ from the interval $[0,0.9]$. These data sets $S_{1 i}, i \in\{1,2,3,4\}$, are as follows:

1. $\delta_{K_{0}}=\delta_{T_{1}}=\delta_{T_{2}}=\delta_{\mu_{1}}=0.0 . \quad 3 . \quad \delta_{K_{0}}=0.6, \delta_{T_{1}}=\delta_{T_{2}}=\delta_{\mu_{1}}=0.9$.

2. $\delta_{K_{0}}=\delta_{T_{1}}=\delta_{T_{2}}=\delta_{\mu_{1}}=0.3$. 4. $\quad \delta_{K_{0}}=\delta_{T_{1}}=\delta_{T_{2}}=\delta_{\mu_{1}}=0.9$.

It should be noted that data set $S_{11}$ corresponds to the mechanical system for which there are no uncertainties of the model parameters. The following data ensembles $S_{2 i}, i \in\{1,2,3,4\}$, of values $h_{0}, N$, and $M$ together with $J=8$ for GFM have been selected for another parameters:

1. $h_{0}=h_{0}^{[1]}, N=N^{[1]}, M=1000$. 3. $h_{0}=h_{0}^{[2]}, N=N^{[2]}, M=2000$.

2. $h_{0}=h_{0}^{[1]}, N=N^{[1]}, M=5000 . \quad 4 . \quad h_{0}=h_{0}^{[2]}, N=N^{[2]}, M=10000$,

where $h_{0}^{[1]}=0.01, h_{0}^{[2]}=0.01, N^{[1]}=120,000, N^{[2]}=240,000$. 


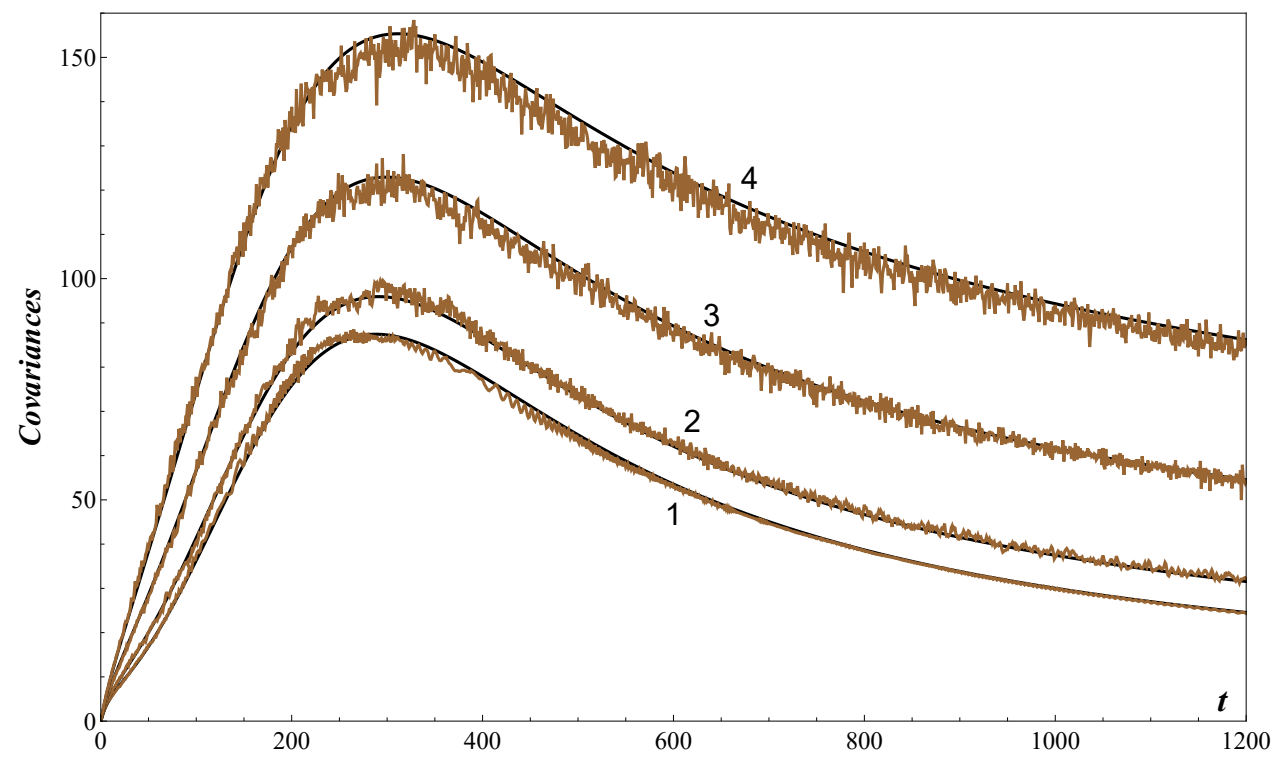

Figure 2: Comparison of GFM and MCM for the function of covariance, $C_{Y_{1} Y_{1}}(t, t)$.

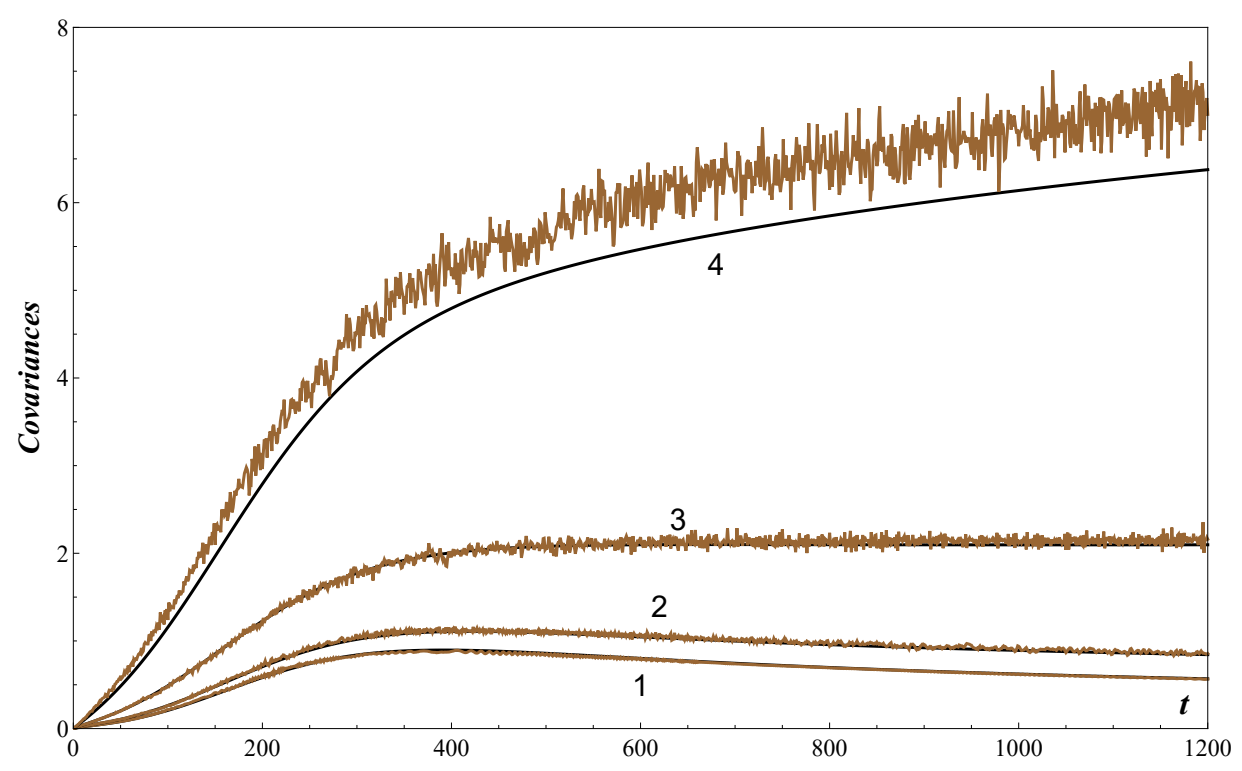

Figure 3: Comparison of GFM and MCM for the function of covariance, $C_{Y_{3} Y_{3}}(t, t)$.

\subsubsection{Figures and explanations}

A part of the first results is presented in Figs. 2 to 7, where smooth and noisy curves show results of GFM and MCM calculations with data ensembles $S_{21}$ and $S_{22}$ respectively. In these figures, additional digits from 1 to 4 refer numbers $i$ of data sets $S_{1 i}$. Figs. 2, 3 show only a portion of curves demonstrating similar re- 


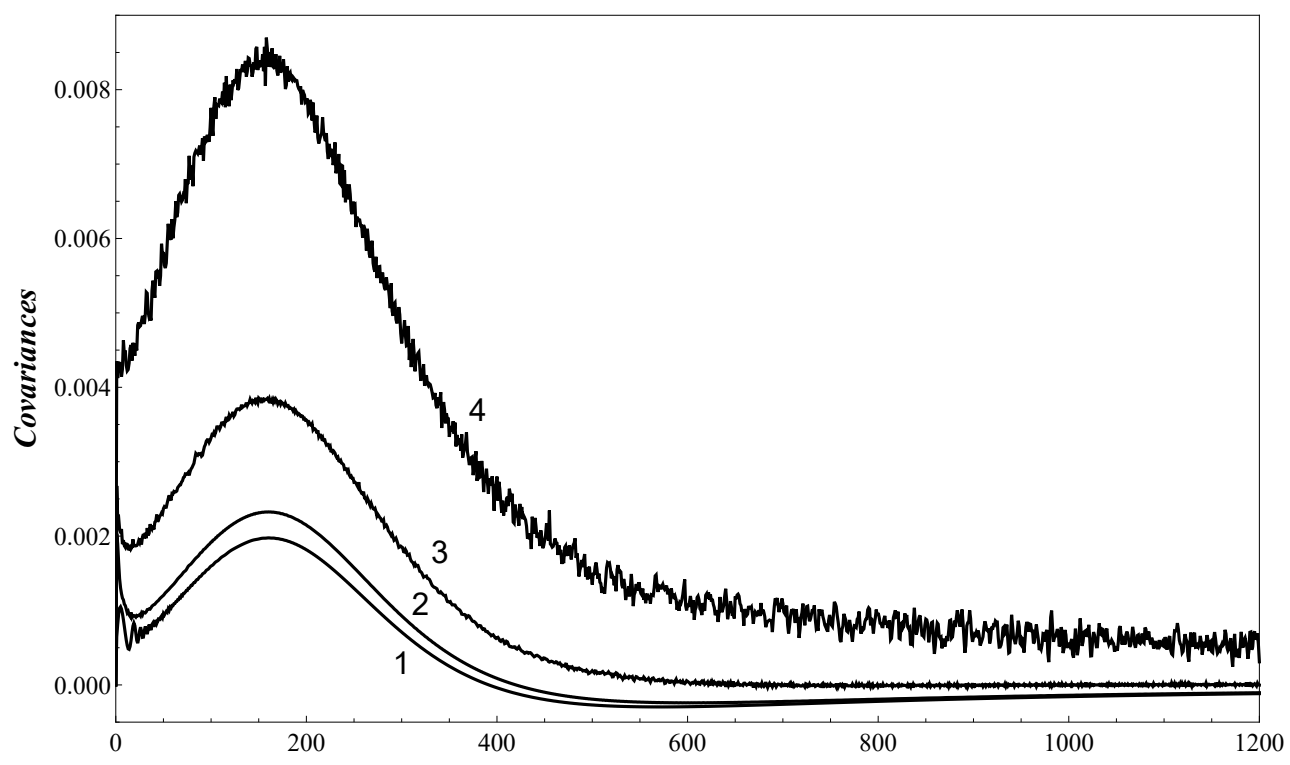

Figure 4: Time history of the functions of covariance, $C_{Y_{3} Y_{1}}(t, t)$.

sults for $C_{Y_{1} Y_{1}}(t, t), C_{Y_{1} Y_{2}}(t, t), C_{Y_{2} Y_{2}}(t, t)$ and $C_{Y_{3} Y_{3}}(t, t), C_{Y_{3} Y_{4}}(t, t), C_{Y_{4} Y_{4}}(t, t)$ obtained by GFM and MCM. The rest part of similar GFM curves, presenting behavior of functions of covariances with small values, are separately shown in Figs. 4-7 because appropriate pulsating MCM estimates are too noisy. As it was said before, the main aim of numerical experiments performed by MCM is a numerical verification of the presented mathematical approach.

The figures show that the time histories, calculated by GFM and MCM, are in a good agreement unless results for data set $S_{14}$. For this data set, differences occur for a part of covariances. Therefore, time histories for this data set have been recalculated by the same methods with reduced time steps $h_{0}$ and increased numbers $M$ of simulations with data ensembles $S_{23}$ and $S_{24}$. But such a numerical strategy has not induced a significant improvement of convergence.

Thus a necessity to resolve a question about quality of the results has forced to produce extra large calculations for data set $S_{14}$ with the following additional parameter ensembles $S_{2 i}, i \in\{5,6\}$ :

5. $h_{0}=0.0005, N=2,400,000, M=20,000$.

6. $h_{0}=0.0001, N=12,000,000, M=100,000$.

These calculations show the following effects:

(i) the time histories computed by GFM for data ensembles $S_{21}$ and $S_{25}$ are differed insignificantly; 


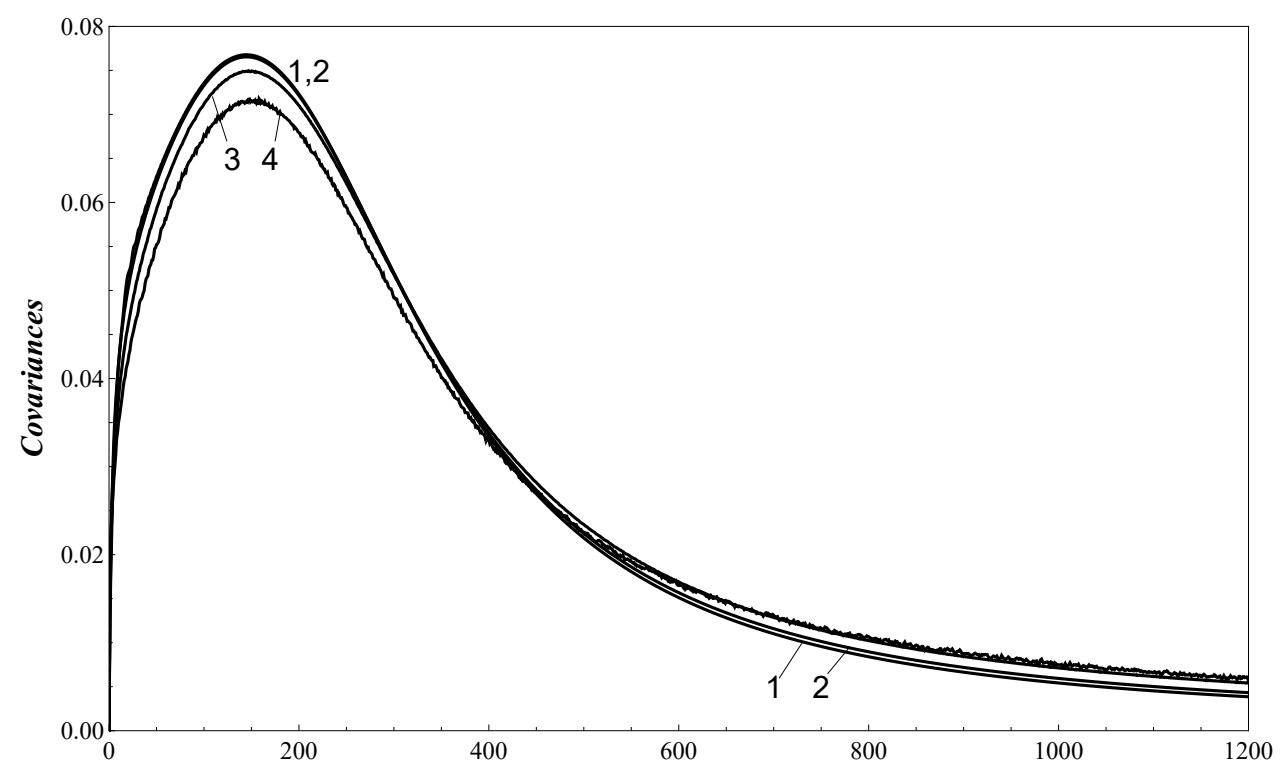

Figure 5: Time history of the functions of covariance, $C_{Y_{3} Y_{2}}(t, t)$.

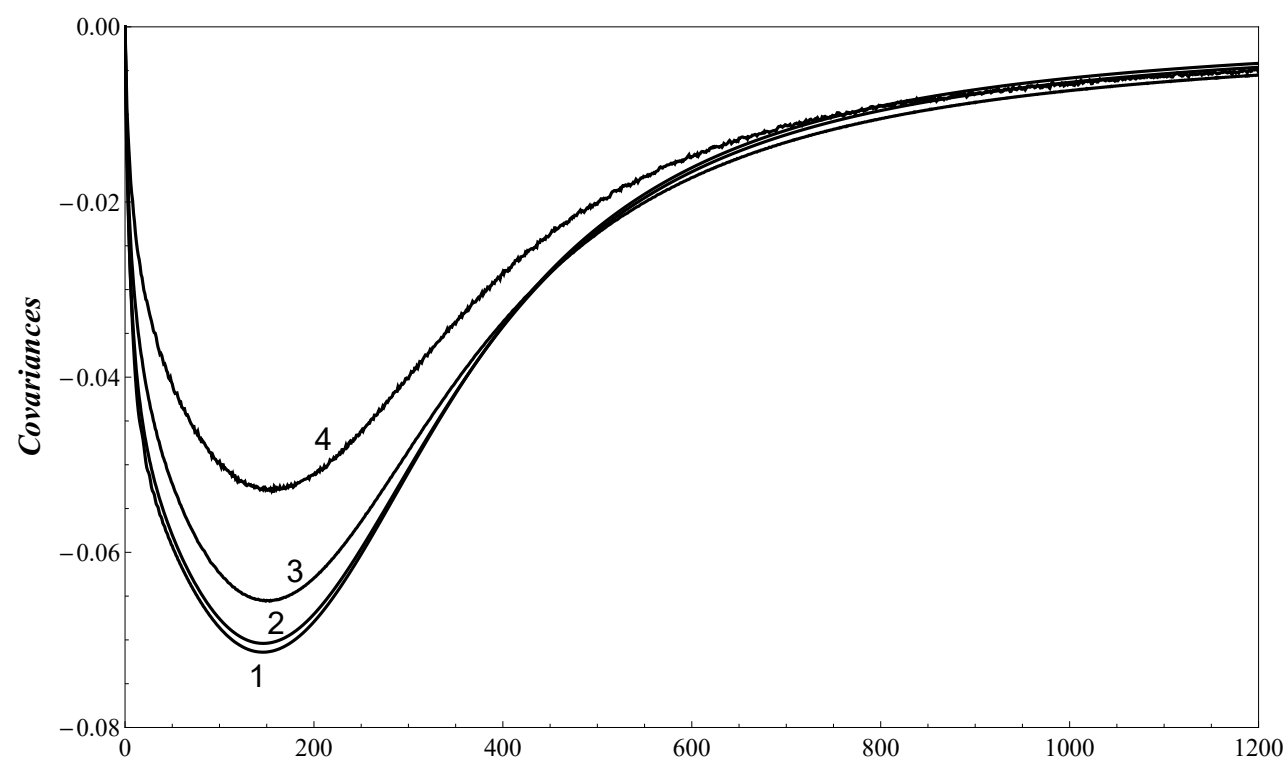

Figure 6: Time history of the functions of covariance, $C_{Y_{4} Y_{1}}(t, t)$.

(ii) if to compare results of calculations for ensembles $S_{22}$ and $S_{26}$, then the changes are more visible for MCM outcomes, namely, amplitudes of fluctuations in time histories of the covariances' estimators have reduced approximately threefold;

(iii) the $C_{Y_{1} Y_{1}}(t, t), C_{Y_{1} Y_{2}}(t, t), C_{Y_{2} Y_{2}}(t, t)$ time histories, calculated for ensem- 


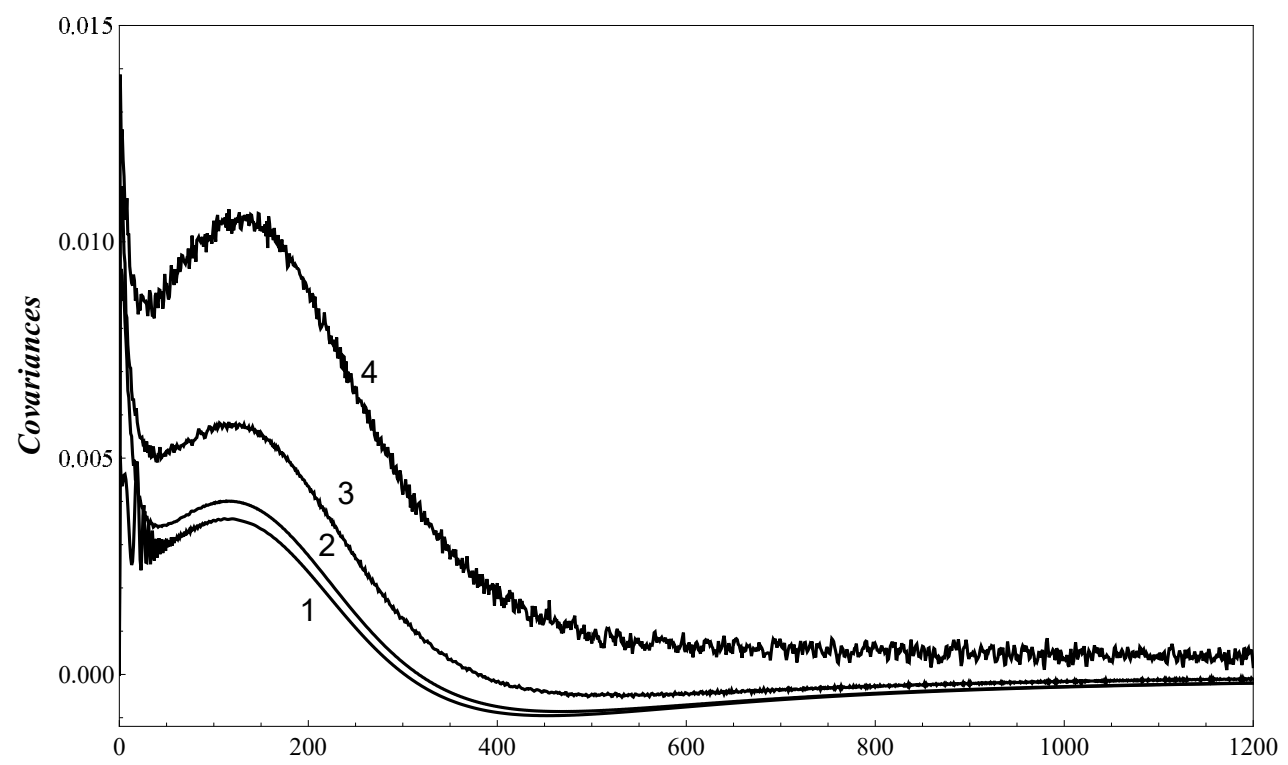

Figure 7: Time history of the functions of covariance, $C_{Y_{4} Y_{2}}(t, t)$.

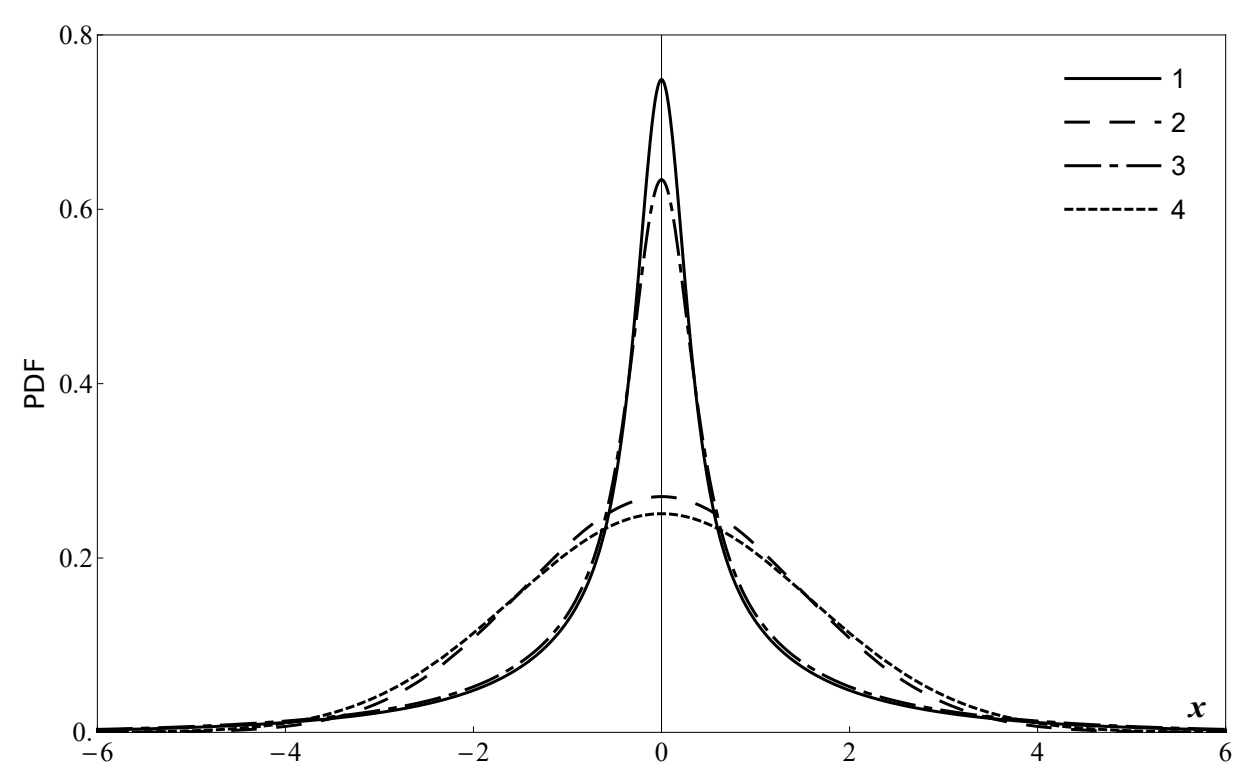

Figure 8: PDFs for $\dot{U}_{3}(1200)$ (curves 1 and 2) and $\dot{U}_{4}(1200)$ (curves 3 and 4).

ble $S_{25}$, are in a good mutual consistency as well as with results of computations for data ensembles $S_{2 i}, i \in\{1,2,3,4\}$;

(iv) the differences between the time histories for GFM and MCM estimates of $C_{Y_{3} Y_{3}}(t, t), C_{Y_{4} Y_{3}}(t, t), C_{Y_{4} Y_{4}}(t, t)$ for data set $S_{14}$ have not significantly decreased.

The results displayed in Figs. 2 to 7 , and produced for another data sets of 
values for $\delta_{K_{0}}, \delta_{T_{1}}, \delta_{\mu_{1}}$ and $\delta_{T_{2}}$, show a prevailing influence of the random variable $A_{K_{0}}$ on the behavior of all the covariances.

The last figure, Fig. 8, clearly demonstrates an action of the random modeling parameters, which is expressed in a non-Gaussian form of response despite the linearity of the dynamical system under investigation and the Gaussian excitation. The PDF curves marked "1" and "3", characterize states of the system with data ensemble 3 at $t=1,200 \mathrm{~s}$. These curves have been built using Eq. (29), where for sample PDFs, the means are equal to zero and the variances coincide with the GFM sample variances. The graphs marked "2" and "4", correspond to appropriate Gaussian PDFs with zero means and variances computed by Eq. (28). The same effect is observed for $\dot{U}_{1}(1200)$ and $\dot{U}_{2}(1200)$.

Computational problems for the data set $S_{14}$ in the MCM analysis of the system can be explained as follows:

1. The system is stochastic and random one.

2. The multiplicative random parameter $A_{K_{0}}$ affects the variable frequency of oscillation of the system. At the beginning of the transition, the frequency changes almost four times for the extreme values of $A_{K_{0}}$. This leads to the necessity of statistical processing of substantially different time histories of $U_{1}(t), \dot{U}_{1}(t), U_{2}(t), \dot{U}_{2}(t)$.

3. Usually MCM schemes are designed without taking into account of random parameters that can render a large effect on time histories of systems under examinations, i.e., these histories form heterogeneous samples.

4. An implementation of MCM processes stochasticity and randomness but an implementation of GFM deals only with randomness. Errors of statistical treatment of randomness can be considered the same for both schemes.

5. The error of a weak approximation of semi-implicit scheme for MCM is $O\left(h_{0}\right)$ and the same for GFM is $O\left(h_{0}^{L}\right)$. Therefore a full error for GFM is substantially less than for MCM.

It should be noted that the results obtained are significantly different when the transformations of pseudo-random numbers are not used. For example, in the last case, we have observed parasitic transitional regimes that didn't exist at the exact adherence to the original formulation of the problem.

The small fluctuations that occur in the results are due to the number of samples used in Monte Carlo simulation method. The relative error induced by such a method is in $1 /$ sqrt(numer of samples) and is independent of the dimension of the vector valued-random parameter. Consequently, such fluctutaions could be reduced in increasing the number of samples. 


\section{Conclusions}

In this paper, we have presented some results of computation for $\mathcal{C}_{Y Y}^{Q}(t, t)$, $t_{0} \leqslant t \leqslant T$, produced by the presented scheme. These results are in a good agreement with outcomes obtained by the Monte Carlo simulation method. If it was necessary to calculate $\mathcal{C}_{Y Y}^{Q}\left(t, t^{\prime}\right), t_{0} \leqslant t^{\prime} \leqslant t \leqslant T$, then the computation scheme proposed should be lightly modified for the use of parallel calculations along layers of $t^{\prime}$, i.e., for $t^{\prime}$ fixed and changing $t>t^{\prime}$.

For a given precision, the method proposed allows for considerably decreasing the computational effort with respect to a global Monte Carlo approach.

It is important to highlight that, in the scheme proposed, the use of the Monte Carlo method is minimal. Moreover, if the vector $Q$ was deterministic, then the Monte Carlo simulation method would not be required.

The proposed scheme is suitable for the analysis of linear SOIDE subjected to an additive noise. In the case of a presence of multiplicative noises, another schemes should be constructed for analyzing such parametric linear SODE and SOIDE.

\section{Acknowledgement}

This research did not receive any specific grant from funding agencies in the public, commercial, or not-for-profit sectors.

\section{Appendix A. Coefficients of the Taylor series for $L=4$}

For $L=4$, the coefficients of the Taylor series in Eq. (22) are given by the following equations:

$$
\begin{gathered}
\Phi_{1}(s, s)=\mathcal{A}(s) \\
\Phi_{2}(s, s)=\mathcal{A}^{\prime}(s)+\mathcal{A}(s) \Phi_{1}(s, s)+\mathcal{B}(s, s) \\
\Phi_{3}(s, s)=\mathcal{A}^{\prime \prime}(s)+\left[2 \mathcal{A}^{\prime}(s)+\mathcal{B}(s, s)\right] \Phi_{1}(s, s) \\
+\mathcal{A}(s) \Phi_{2}(s, s)+\left.\left(2 \frac{\partial}{\partial s}+\frac{\partial}{\partial \tau}\right) \mathcal{B}(s, \tau)\right|_{\tau=s} \\
\Phi_{4}(s, s)=\mathcal{A}^{\prime \prime \prime}(s)+\left[3 \mathcal{A}^{\prime \prime}(s)+\left.\left(3 \frac{\partial}{\partial s}+2 \frac{\partial}{\partial \tau}\right) \mathcal{B}(s, \tau)\right|_{\tau=s}\right] \Phi_{1}(s, s) \\
+3\left[\mathcal{A}^{\prime}(s)+\mathcal{B}(s, s)\right] \Phi_{2}(s, s)+\mathcal{A}(s) \Phi_{3}(s, s) \\
+\left.\left(3 \frac{\partial^{2}}{\partial s^{2}}+3 \frac{\partial^{2}}{\partial s \partial \tau}+\frac{\partial^{2}}{\partial \tau^{2}}\right) \mathcal{B}(s, \tau)\right|_{s=\tau}
\end{gathered}
$$




\section{References}

[1] V. Bolotin, Random Vibrations of Elastic Systems, Springer Science + Business Media, Dordrecht, 1984.

[2] P. Krée, C. Soize, Mathematics of Random Phenomena: Random Vibrations of Mechanical Structures, D. Reidel Publishing Company, Dordrecht, 1986.

[3] Y. Lin, G. Cai, Probabilistic Structural Dynamics, McGraw-Hill, New York, 2004.

[4] G. Schueller, H. Pradlwarter, Uncertain linear systems in dynamics: Retrospective and recent developments by stochastic approaches, Engineering Structures 31 (11) (2009) 2507-2517.

[5] C. Soize, Uncertainty Quantification. An Accelerated Course with Advanced Applications in Computational Engineering, Springer, New York, 2017.

[6] T. Soong, M. Grigoriu, Random Vibrations of Structural and Mechanical Systems, Prentice Hall, New Jersey, 1993.

[7] C. Soize, I. Poloskov, Time-domain formulation in computational dynamics for linear viscoelastic media with model uncertainties and stochastic excitation, Computers and Mathematics with Applications 64 (11) (2012) 35943612 .

[8] M. Leitman, G. Fisher, The linear theory of viscoelasticity, in: C. Truesdell (Ed.), Encyclopedia of Physics, Vol. VI a/3, Springer-Verlag, Berlin and Heidelberg and New York, 1973, pp. 1-123.

[9] V. Potapov, Stability of Stochastic Elastic and Viscoelastic Systems, John Wiley and Sons, Chichester, 1999.

[10] V. Zvyagin, V. Orlov, A weak solvability of a system of thermoviscoelasticity for the Jeffreys model, Russian Mathematics (Iz. VUZ) 57 (9) (2013) 53-57.

[11] A. Drozdov, V. Kolmanovskii, P. Nistri, P. Zecca, Stability of nonhomogeneous aging viscoelastic bodies under dynamic loading, Nonlinear Anal.: Theory, Methods and Applications 24 (9) (1995) 1361-1375.

[12] A. Pani, V. Thomée, L. Wahlbin, Numerical methods for hyperbolic and parabolic integro-differential equations, J. Integral Equations Appl. 4 (4) (1992) 533-584. 
[13] A. Khani, M. Moghadam, S. Shahmorad, Approximate solution of the system of non-linear volterra integro-differential equations, Computational Methods in Applied Mathematics 8 (1) (2008) 77-85.

[14] J. Day, Note on the numerical solution of integro-differential equations, The Computer Journal 9 (4) (1967) 394-395.

[15] H. Nguyen, T. Herdman, E. Cliff, Approximations for a class of Volterra integro-differential equations, Mathematical and Computer Modelling 42 (5-6) (2005) 659-672.

[16] V. Potapov, Nonlinear vibrations and stability of elastic and viscoelastic systems under random stationary loads, Mechanics of Solids 46 (3) (2011) 444454.

[17] M. Chaikovskii, L. Yanovich, On numerical finding of correlation functions for solution of systems of linear integro-differential equations with random perturbations in right-hand parts, Russian Differential Equations 23 (2) (1987) 328-338.

[18] S. Chang, On certain extrapolation methods for the numerical solution of integro-differential equations, Mathematics of Computation 39 (159) (1982) $165-171$.

[19] T. Lin, Y. Lin, M. Rao, S. Zhang, Petrov-Galerkin methods for linear Volterra integro-differential equations, SIAM Journal on Numerical Analysis 38 (3) (2001) 937-963.

[20] D. Han, X. Shang, Numerical solution of integro-differential equations by using CAS wavelet operational matrix of integration, Applied Mathematics and Computation 194 (2) (2007) 460-466.

[21] K. Jalaei, M. Zarebnia, M. Chalaki, Development of the Sinc method for nonlinear integro-differential equations, Austr. J. of Basic and Applied Sciences 4 (11) (2010) 5508-5515.

[22] I. Poloskov, On calculation of the first moments for linear integro-differential systems with parametric excitations, in: Problems of Mechanics and Control: Nonlinear Dynamical Systems, Vol. 38, Perm State Univ., Perm, 2006, pp. 133-142.

[23] A. Goldfine, Taylor series methods for the solution of Volterra integral and integro-differential equations, Mathematics of Computation 31 (139) (1977) 691-707. 
[24] S. Hu, V. Lakshmikantham, Monotone iterative technique for integrodifferential equations, in: V. Korolyuk (Ed.), Asymptotic Methods of Mathematical Physics, Naukova Dumka, Kiev, 1988, pp. 263-270.

[25] V. Nguyen, Asymptotic method of analysis of multiple-frequency vibrations in quasi-linear systems of integro-differential equations of the second order, Ukrainian Mathematical Journal 29 (3) (1977) 404-410.

[26] H. Brunner, High-order methods for the numerical solution of Volterra integro-differential equations, Journal of Computational and Applied Mathematics 15 (3) (1986) 301-309.

[27] S. Grossman, R. Miller, Perturbation theory for Volterra integrodifferential systems, Journal of Differential Equations 8 (3) (1970) 457-474.

[28] I. Poloskov, Compound program packages and a nonlinear random fluctuations analysis, in: V. Weispfenning, B. Trager (Eds.), Proc. of the 1998 Intern. Symp. on Symbolic and Algebraic Computation (ISSAC-98), ACM Press, New York, 1998, pp. 70-75.

[29] I. Poloskov, On a method for approximate analysis of linear stochastic integro-differential systems, Russian Differential Equations 41 (9) (2005) 1349-1352.

[30] B. Coleman, On the thermodynamics, strain impulses and viscoelasticity, Arch. Rational Mech. Anal. 17 (1964) 230-254.

[31] M. Gurtin, I. Herrera, On dissipation inequalities and linear viscoelasticity, Quart. Appl. Math. 23 (3) (1965) 235-245.

[32] A. Drozdov, A constitutive model in thermoviscoelasticity, Mechanics Research Communications 23 (5) (1996) 543-548. 